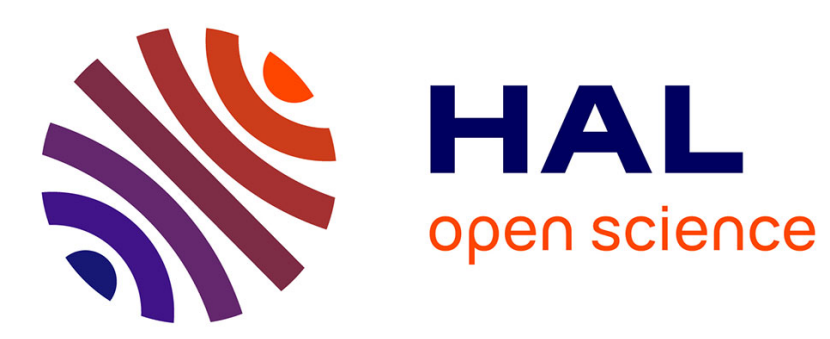

\title{
Error estimates for the convergence of a finite volume discretization of convection-diffusion equations
}

\author{
Jerome Droniou
}

\section{To cite this version:}

Jerome Droniou. Error estimates for the convergence of a finite volume discretization of convection-diffusion equations. Journal of Numerical Mathematics, 2003, 11 (1), pp.1-32. 10.1515/156939503322004873 . hal-00018749

\section{HAL Id: hal-00018749 \\ https://hal.science/hal-00018749}

Submitted on 8 Feb 2006

HAL is a multi-disciplinary open access archive for the deposit and dissemination of scientific research documents, whether they are published or not. The documents may come from teaching and research institutions in France or abroad, or from public or private research centers.
L'archive ouverte pluridisciplinaire HAL, est destinée au dépôt et à la diffusion de documents scientifiques de niveau recherche, publiés ou non, émanant des établissements d'enseignement et de recherche français ou étrangers, des laboratoires publics ou privés. 


\title{
Error estimates for the convergence of a finite volume dis- cretization of convection-diffusion equations
}

\author{
J. DRONIOU*
}

Received 04 October, 2002

Communicated by R. Lazarov

Received in revised form 23 December, 2002

\begin{abstract}
We study error estimates for a finite volume discretization of an elliptic equation. We prove that, for $s \in[0,1]$, if the exact solution belongs to $H^{1+s}$ and the right-hand side is $f+\operatorname{div}(G)$ with $f \in L^{2}$ and $G \in\left(H^{s}\right)^{N}$, then the solution of the finite volume scheme converges in discrete $H^{1}$ norm to the exact solution, with a rate of convergence of order $h^{s}$ (where $h$ is the size of the mesh).
\end{abstract}

Keywords: Convection-diffusion equations, Finite Volume, convergence rate, interpolation

\section{INTRODUCTION}

\subsection{The problem}

Let $\Omega$ be a polygonal open subset of $\mathbb{R}^{N}$ ( $N=2$ or 3 ). We study a finite volume discretization of

$$
\begin{cases}-\Delta \bar{u}+\operatorname{div}(\mathbf{v} \bar{u})+b \bar{u}=f+\operatorname{div}(G) & \text { in } \Omega \\ \bar{u}=0 & \text { on } \partial \Omega\end{cases}
$$

where $\mathbf{v} \in(C(\bar{\Omega}))^{N}, b \in L^{\infty}(\Omega)$ is nonnegative, $f \in L^{2}(\Omega)$ and $G \in\left(H^{s}(\Omega)\right)^{N}$ with $s \in[0,1]$ (if $s=0, H^{0}(\Omega)$ is to be understood as $L^{2}(\Omega)$ ).

The solution to (1.1) is taken in a weak sense as in [6], that is to say $\bar{u} \in H_{0}^{1}(\Omega)$ and the partial differential equation is satisfied in the distributional sense.

Finite volume methods have been widely used to approximate the solutions of convection-diffusion equations (see e.g. [9], [10], [8]...). The convergence of the approximations is well-known (see e.g. [9] (Theorem 9.1)) and some error estimates in the $H^{2}$ framework have been obtained in [10] (Theorem 3.2).

These schemes have mainly been considered when the right-hand side of the elliptic equation belongs to $L^{2}(\Omega)$ (i.e. $G=0$ in (1.1)); but, recently, [8] has presented a finite volume scheme capable of handling (1.1) with any $G \in\left(L^{2}(\Omega)\right)^{N}$. The case

\footnotetext{
*Département de Mathématiques, CC 051, Université Montpellier II, Place Eugène Bataillon, 34095 Montpellier cedex 5, France. email: droniou@math. univ-montp2. fr
} 
$G \in\left(H^{1}(\Omega)\right)^{N}$ being (roughly speaking) the $H^{2}$ framework studied in [10], it seems natural to hope, via interpolation techniques, for error estimates when $G$ belongs to intermediate spaces between $L^{2}(\Omega)$ and $H^{1}(\Omega)$ (these estimates are well-known for the finite element methods, see for example [3] (Theorem 5.1)). We prove here such error estimates, thus filling a gap between finite volume methods and finite element methods.

It can be interesting to notice that, in the case $N=2$ and for "finite volume element" schemes (which are somewhat a mixing between the finite element methods and the finite volume methods, and are different from the ones we present here), some error estimates in the $H^{1+s}$ framework have been obtained in [5] (Theorem 4.1, p. 176), when $s \in] 1 / 2,1]$.

We also emphasize on a noticeable feature of (1.1): its non-coercivity. It is not supposed that $\frac{1}{2} \operatorname{div}(\mathbf{v})+b \geqslant 0$, so that the bilinear form associated to (1.1) may be non-coercive. However, under the sole hypotheses stated after (1.1), existence and uniqueness of a solution to this equation is known (see [6] (Theorem 2.1)). The $a$ priori estimates on this equation and its discretization are harder to obtain than in the coercive case, but the techniques that give such estimates are now well-known (see [6], [8]).

In the following subsection, we present the finite volume scheme used to discretize (1.1); this scheme is in fact a simplified version of the one presented in [8] (simplified because we take into account the additional regularity we have on $\mathbf{v}$ with respect to [8]). In Section 2, we state the main result concerning estimates on the difference between the approximate solution and the exact solution; notice that we have to use a different discretization of the exact solution than in [10], because we do not only intend to study the case when this solution belongs to $H^{2}(\Omega)$, but also when it belongs to $H^{1+s}(\Omega)$ for $s \in[0,1]$; hence we cannot, in contrary to [10], discretize the solution by taking its values on points. In Section 3, we study the case when the solution belongs to $H^{1}$; in this case, the "error estimate" reduces to a bound in $\mathscr{O}(1)$. In Section 4 , we prove a $\mathscr{O}(h)$ convergence when the exact solution belongs to $H^{2}(\Omega)$ and $G$ belongs to $\left(H^{1}(\Omega)\right)^{N}$; note that, since our discretization of the solution is not the same as in [10], we cannot directly refer to this paper and we must re-make the whole work (moreover, our scheme is different to the one presented in [10], because of the presence of $G$ ); in particular, it appears through this study that the way we discretize the solution is crucial to obtain good error estimates. In Section 5, we use the results of Sections 3 and Sections 4 to prove, via interpolation results, the theorem stated in Section 2. Section 6 presents some numerical results, and Section 7 is an appendix which gathers some technical lemmas useful throughout the paper.

\subsection{Definition of the scheme}

We use meshes of $\Omega$ similar to the ones presented in [9].

Definition 1.1. An admissible mesh $\mathscr{T}$ of $\Omega$ is a finite family of polygonal open 
convex subsets of $\Omega$ (the "control volumes"), together with a finite family $\mathscr{E}$ of disjoint subsets of $\bar{\Omega}$ consisting in non-empty open convex subsets of affine hyperplanes (the "edges") and a family $\mathscr{P}=\left(x_{K}\right)_{K \in \mathscr{T}}$ of points in $\Omega$ such that

i) $\bar{\Omega}=\bigcup_{K \in \mathscr{T}} \bar{K}$,

ii) each $\sigma \in \mathscr{E}$ is contained in $\partial K$ for some $K \in \mathscr{T}$,

iii) by denoting $\mathscr{E}_{K}=\{\sigma \in \mathscr{E} \mid \sigma \subset \partial K\}, \partial K=\cup_{\sigma \in \mathscr{E}_{K}} \bar{\sigma}$ for all $K \in \mathscr{T}$,

iv) for all $K \neq L$ in $\mathscr{T}$, either the $(N-1)$-dimensional measure of $\bar{K} \cap \bar{L}$ is null, or $\bar{K} \cap \bar{L}=\bar{\sigma}$ for some $\sigma \in \mathscr{E}$, that we denote then $\sigma=K \mid L$,

v) for all $K \in \mathscr{T}, x_{K} \in K$,

vi) for all $\sigma=K \mid L \in \mathscr{E}$, the line $\left(x_{K}, x_{L}\right)$ intersects and is orthogonal to $\sigma$,

vii) for all $\sigma \in \mathscr{E}, \sigma \subset \partial \Omega \cap \partial K$, the line which is orthogonal to $\sigma$ and going through $x_{K}$ intersects $\sigma$.

If $K \in \mathscr{T}, h_{K}$ denotes the diameter of $K$; the size of $\mathscr{T}$ is $h_{\mathscr{T}}=\sup _{K \in \mathscr{T}} h_{K}$. The unit normal to $\sigma \in \mathscr{E}_{K}$ outward to $K$ is denoted by $\mathbf{n}_{K, \sigma}$.

We define $\mathscr{E}_{\text {int }}=\{\sigma \in \mathscr{E} \mid \sigma \not \subset \partial \Omega\}$ (interior edges) and $\mathscr{E}_{\text {ext }}=\mathscr{E} \backslash \mathscr{E}$ int. We denote by $m$ the $(N-1)$-dimensional measure on the edges of the mesh so that, if $\sigma \in \mathscr{E}, m(\sigma)$ is the $(N-1)$-dimensional measure of $\sigma$. If $\sigma=K \mid L \in \mathscr{E}$ int,$d_{\sigma}$ is the Euclidean distance between the points $\left(x_{K}, x_{L}\right)$ and $d_{K, \sigma}$ denotes the distance between $x_{K}$ and $\sigma$; if $\sigma \in \mathscr{E}_{\text {ext }} \cap \mathscr{E}_{K}, d_{\sigma}=d_{K, \sigma}$ is the distance between $x_{K}$ and $\sigma$.

If $K \in \mathscr{T}$ and $\sigma \in \mathscr{E}_{K}$, the "half-diamond" $\triangle_{K, \sigma}$ is defined by $\triangle_{K, \sigma}=\left\{t x_{K}+\right.$ $(1-t) x, t \in] 0,1[, x \in \sigma\}$ (the convex hull of $\left\{x_{K}\right\} \cup \sigma$ ). We notice that $\left|\triangle_{K, \sigma}\right|=$ $m(\sigma) d_{K, \sigma} / N$ (where $|\cdot|$ denotes the Lebesgue measure in $\mathbb{R}^{N}$ ).

We also make the following hypotheses on the meshes:

$$
\begin{gathered}
\exists \zeta>0 \text { such that } \forall K \in \mathscr{T}, d_{K, \sigma} \geqslant \zeta d_{\sigma}, \\
\exists \alpha>0 \text { such that } \forall K \in \mathscr{T}, B\left(x_{K}, \alpha h_{K}\right) \subset K, \\
\exists M>0 \text { such that } \forall K \in \mathscr{T}, \operatorname{card}\left(\mathscr{E}_{K}\right) \leqslant M
\end{gathered}
$$

$(B(x, \eta)$ denotes the ball of center $x$ and radius $\eta)$.

Hypothesis (1.2) is classical when discrete Sobolev inequalities are needed. These inequalities are useful in a priori estimates on the scheme (to control the convective term of the equation), which appear in [8] or [7]. Here, we will directly use the results of [7], hence the need for (1.2) will not be glaring. Notice that if $\mathbf{v}=0$ (or $\mathbf{v}$ is regular and $\operatorname{div}(\mathbf{v}) \geqslant 0$ ), then Hypothesis (1.2) can be dropped.

(1.3) and (1.4) appear in [9] for the same kind of results that we present here (see Remarks 3.1 and 4.3). 
Remark 1.1. As an example of admissible meshes, we can take regular uniform meshes as in Section 6, but also triangular meshes (provided that all angles of the triangles are less than $\pi / 2$ - this can be relaxed, see Example 9.1 in [9]) and most of Vorono 1 i meshes (see Example 9.2 in [9]).

The finite volume discretization is obtained by an integration of the equation on a control volume $K$ : with some integrates by parts, we formally obtain

$$
\begin{aligned}
\sum_{\sigma \in \mathscr{E}_{K}}-\int_{\sigma} \nabla \bar{u} \cdot \mathbf{n}_{K, \sigma} d m+ & \sum_{\sigma \in \mathscr{E}_{K}} \int_{\sigma} \bar{u} \mathbf{v} \cdot \mathbf{n}_{K, \sigma} d m+\int_{K} b \bar{u} \\
& =\int_{K} f+\sum_{\sigma \in \mathscr{E}_{K}} \int_{\sigma} G \cdot \mathbf{n}_{K, \sigma} d m .
\end{aligned}
$$

To discretize this equation, we define, for $K \in \mathscr{T}$ and $\sigma \in \mathscr{E}_{K}$,

$$
\begin{aligned}
& v_{K, \sigma}=\left(\frac{1}{m(\sigma)} \int_{\sigma} \mathbf{v}(\xi) d m(\xi)\right) \cdot \mathbf{n}_{K, \sigma}, \quad b_{K}=\frac{1}{|K|} \int_{K} b(x) d x, \\
& f_{K}=\frac{1}{|K|} \int_{K} f(x) d x \quad \text { and } \quad G_{K, \sigma}=\left(\frac{1}{\left|\triangle_{K, \sigma}\right|} \int_{\triangle_{K, \sigma}} G(x) d x\right) \cdot \mathbf{n}_{K, \sigma},
\end{aligned}
$$

which are approximate values of $\mathbf{v} \cdot \mathbf{n}_{K, \sigma}$ and $G \cdot \mathbf{n}_{K, \sigma}$ on $\sigma$, and of $b$ and $f$ on $K$.

Then, letting $u_{K}$ and $u_{\sigma}$ be approximate values of $\bar{u}$ on $K$ and $\sigma$, the finite volume discretization of (1.1) is written

$$
\begin{gathered}
\forall K \in \mathscr{T}, \sum_{\sigma \in \mathscr{E}_{K}} F_{K, \sigma}+\sum_{\sigma \in \mathscr{E}_{K}} m(\sigma) v_{K, \sigma} u_{\sigma,+}+|K| b_{K} u_{K} \\
=|K| f_{K}+\sum_{\sigma \in \mathscr{E}_{K}} m(\sigma) G_{K, \sigma}, \\
\forall K \in \mathscr{T}, \forall \sigma \in \mathscr{E}_{K}, \quad F_{K, \sigma}=-\frac{m(\sigma)}{d_{K, \sigma}}\left(u_{\sigma}-u_{K}\right), \\
\forall \sigma=K \mid L \in \mathscr{E}_{\text {int }}, \quad F_{K, \sigma}-m(\sigma) G_{K, \sigma}=-\left(F_{L, \sigma}-m(\sigma) G_{L, \sigma}\right), \\
\forall \sigma \in \mathscr{E}_{\mathrm{ext}}, \quad u_{\sigma}=0, \\
\forall \sigma=K \mid L \in \mathscr{E}_{\text {int }}, \quad u_{\sigma,+}=u_{K} \text { if } v_{K, \sigma} \geqslant 0, \quad u_{\sigma,+}=u_{L} \text { otherwise, } \\
\forall \sigma \in \mathscr{E}_{\mathrm{ext}} \cap \mathscr{E}_{K}, \quad u_{\sigma,+}=u_{K} \text { if } v_{K, \sigma} \geqslant 0, \quad u_{\sigma,+}=0 \text { otherwise }
\end{gathered}
$$

$\left(F_{K, \sigma}\right.$ is of course a discretization of $\left.-\int_{\sigma} \nabla u \cdot \mathbf{n}_{K, \sigma} d m\right)$.

Remark 1.2. (1.8) is a classical upwind choice of the discretization of the convection term $\operatorname{div}(\mathbf{v} \bar{u})$ (see [9], p. 766). This choice brings stability to the scheme and allows unconditional a priori estimates (see [7] (Proposition 3.2, p. 72)); notice however that, since our problem is non-coercive (i.e. $\operatorname{div}(\mathbf{v})$ is not supposed nonnegative), the maximum principle on the scheme (1.5)—(1.8) is not known. 
The scheme (1.5) - (1.8) is not exactly the same as in [8], because $\mathbf{v}$ has not been discretized the same way. In fact, in [8], $\mathbf{v}$ is less regular, so it must be discretized using mean values on $\triangle_{K, \sigma}$, not on $\sigma$; to obtain error estimates, we must assume here that $\mathbf{v}$ is more regular than in [8], so it seems more natural (and easier!), since the regularity of $\mathbf{v}$ allows it, to consider mean values of $\mathbf{v}$ on $\sigma$ rather than $\triangle_{K, \sigma}$.

In fact, the unknowns $\left(u_{\sigma}\right)_{\sigma \in \mathscr{E}}$ in (1.5)-(1.8) can be immediately eliminated thanks to (1.7), and (1.5) - (1.8) reduces thus to a system with unknowns $\left(u_{K}\right)_{K \in \mathscr{T}}$, which reads

$$
\begin{aligned}
& \forall K \in \mathscr{T}, \sum_{\sigma \in \mathscr{E}_{K}} \frac{m(\sigma)}{d_{\sigma}}\left(u_{K}-u_{L}\right)+\sum_{\sigma \in \mathscr{E}_{K}} m(\sigma) v_{K, \sigma} u_{\sigma,+}+|K| b_{K} u_{K} \\
& \quad=|K| f_{K}+\sum_{\sigma \in \mathscr{E}_{K}} m(\sigma)\left(\frac{d_{K, \sigma}}{d_{\sigma}} G_{K, \sigma}-\frac{d_{L, \sigma}}{d_{\sigma}} G_{L, \sigma}\right) \\
& \text { (where } \sigma=K \mid L \text { if } \sigma \in \mathscr{E}_{K} \cap \mathscr{E}_{\text {int }} \text { and } \\
& \left.u_{L}=d_{L, \sigma}=G_{L, \sigma}=0 \text { if } \sigma \in \mathscr{E}_{K} \cap \mathscr{E}_{\mathrm{ext}}\right), \\
& \forall \sigma=K \mid L \in \mathscr{E}_{\text {int }}, \quad u_{\sigma,+}=u_{K} \text { if } v_{K, \sigma} \geqslant 0, \quad u_{\sigma,+}=u_{L} \text { otherwise, } \\
& \forall \sigma \in \mathscr{E}_{\mathrm{ext}} \cap \mathscr{E}_{K}, \quad u_{\sigma,+}=u_{K} \text { if } v_{K, \sigma} \geqslant 0, \quad u_{\sigma,+}=0 \text { otherwise. }
\end{aligned}
$$

A priori estimates on (1.9) - (1.10) are then direct consequences of Proposition 3.2, p.72, in [7] (see (3.5) in the proof of Corollary 3.1 below). These estimates show that the linear system (1.9)-(1.10) is invertible and thus that there exists a unique solution $\left(u_{K}\right)_{K \in \mathscr{T}}$ to this system.

Remark 1.3. In [12], some non-coercive problems are also handled from a numerical point of view. However, the scheme used (namely a Finite Volume Element scheme) is not a Finite Volume scheme as the one we present here, and the regularity on the datas ( $\mathbf{v}$ and the mesh) are quite stronger. Moreover, in this reference, the existence of a solution to the Finite Volume Element scheme is obtained only for $h_{\mathscr{T}}$ small enough.

\section{STATEMENT OF THE MAIN RESULT}

The discretization $\bar{u}_{\mathscr{T}}=\left(\bar{u}_{K}\right)_{K \in \mathscr{T}}$ of the exact solution $\bar{u}$ on an admissible mesh $\mathscr{T}$ is defined by

$$
\forall K \in \mathscr{T}, \bar{u}_{K}=\frac{1}{\left|B\left(x_{K}, \alpha h_{K}\right)\right|} \int_{B\left(x_{K}, \alpha h_{K}\right)} \bar{u}(x) d x .
$$

A more natural way to discretize the exact solution would perhaps be to take the mean value of $\bar{u}$ on each cell $K$. This is not a problem when handling the $H^{1}$ case (see Remark 3.1), but such a discretization would lead to bad constitency errors in the $H^{2}$ case (see Remark 4.1).

If $\mathscr{T}$ is an admissible mesh, we identify the elements $\left(v_{K}\right)_{K \in \mathscr{T}} \in \mathbb{R}^{\operatorname{Card}(\mathscr{T})}$ to functions $v_{\mathscr{T}}$ defined a.e. on $\Omega$ and constant on each cell $K \in \mathscr{T}$. We denote by 
$X(\mathscr{T})$ this space of functions, and it is endowed with the discrete $H^{1}$-norm (which is a natural norm when considering Finite Volume discretizations of elliptic equations):

$$
\left\|v_{\mathscr{T}}\right\|_{1, \mathscr{T}}=\left(\sum_{\sigma \in \mathscr{E}} \frac{m(\sigma)}{d_{\sigma}}\left(D_{\sigma} v_{\mathscr{T}}\right)^{2}\right)^{1 / 2}
$$

where $D_{\sigma} v_{\mathscr{T}}=\left|v_{K}-v_{L}\right|$ if $\sigma=K \mid L \in \mathscr{E}_{\text {int }}$ and $D_{\sigma} v_{\mathscr{T}}=\left|v_{K}\right|$ if $\sigma \in \mathscr{E}_{K} \cap \mathscr{E}_{\text {ext }}$.

In the sequel, all the estimates will be made through this norm. Notice that $\|\cdot\|_{L^{2}(\Omega)} \leqslant \operatorname{diam}(\Omega)\|\cdot\|_{1, \mathscr{T}}$ on $X(\mathscr{T})$ (see [9] (Lemma 9.1) for a proof of this), so that an estimate in $X(\mathscr{T})$ gives a similar estimate in $L^{2}(\Omega)$.

The main result of this paper is the following theorem.

Theorem 2.1. We suppose that $N=2$ or that $\Omega$ is convex; we also assume that $\operatorname{div}(\mathbf{v}) \in L^{2}(\Omega)$. Let $s \in[0,1]$ and $\mathscr{T}$ be an admissible mesh which satisfies Hypotheses (1.2), (1.3) and (1.4). Then there exists $C$ only depending on $\left(\Omega,\|\mathbf{v}\|_{\left(L^{\infty}(\Omega)\right)^{N}},\|b\|_{L^{\infty}(\Omega)}, \zeta, \alpha, M\right)$ such that, if $G \in\left(H^{s}(\Omega)\right)^{N}$ and if the solution $\bar{u}$ to (1.1) belongs to $H^{1+s}(\Omega)$, we have

$$
\left\|\bar{u}_{\mathscr{T}}-u_{\mathscr{T}}\right\|_{1, \mathscr{T}} \leqslant C\left(\|\bar{u}\|_{H^{1+s}(\Omega)}+\|G\|_{\left(H^{s}(\Omega)\right)^{N}}+\|f\|_{L^{2}(\Omega)}\right) h_{\mathscr{T}^{s}}
$$

where $\bar{u}_{\mathscr{T}}=\left(\bar{u}_{K}\right)_{K \in \mathscr{T}}$ is defined by (2.1) and $u_{\mathscr{T}}=\left(u_{K}\right)_{K \in \mathscr{T}}$ is the solution to (1.9)-(1.10).

Remark 2.1. The hypotheses " $N=2$ or $\Omega$ is convex" and " $\operatorname{div}(\mathbf{v}) \in L^{2}(\Omega)$ " are technical hypotheses useful to identify interpolation spaces (see the proof of Theorem 2.1 and Subsection 7.2). In fact, we believe that these hypotheses are not necessary to compute the interpolation spaces that appear in our work, but we have found no result in interpolation literature that allows to get rid of them (for example, to be able to handle non-convex polygonal open sets in dimension 2, we use [2] whose generalization to $N=3$ does not seem easy at all).

It is also to be noticed that these hypotheses are useless if $s=1$ (see Theorem 4.1).

Remark 2.2. Notice that, with our hypotheses, $\Omega$ can be a non-convex polygonal open set of $\mathbb{R}^{2}$, so that assuming $G \in\left(H^{s}(\Omega)\right)^{N}$ does not necessarily implies $\bar{u} \in H^{1+s}(\Omega)$.

\section{THE $H^{1}$ FRAMEWORK}

Proposition 3.1. If $\mathscr{T}$ is an admissible mesh which satisfies Hypotheses (1.3) and (1.4), there exists $C$ only depending on $(N, \alpha, M)$ such that

$$
\left\|\bar{u}_{\mathscr{T}}\right\|_{1, \mathscr{T}} \leqslant C|| \bar{u} \|_{H_{0}^{1}(\Omega)} .
$$


Remark 3.1. In [9] (Lemma 9.4), a similar result is proved (also using Hypotheses (1.3) and (1.4)) with $\bar{u}_{K}$ replaced by the mean value of $\bar{u}$ on $K$.

Proof of Proposition 3.1. Let $K \in \mathscr{T}$ and $\sigma \in \mathscr{E}_{K}$. We have

$$
\begin{aligned}
& \left|\frac{1}{\left|B\left(x_{K}, \alpha h_{K}\right)\right|} \int_{B\left(x_{K}, \alpha h_{K}\right)} \bar{u}(x) d x-\frac{1}{m(\sigma)} \int_{\sigma} \bar{u}(\xi) d m(\xi)\right| \\
& \leqslant \quad\left|\frac{1}{\left|B\left(x_{K}, \alpha h_{K}\right)\right|} \int_{B\left(x_{K}, \alpha h_{K}\right)} \bar{u}(x) d x-\frac{1}{\left|\triangle_{K, \sigma}\right|} \int_{\triangle_{K, \sigma}} \bar{u}(y) d y\right| \\
& \quad+\left|\frac{1}{\left|\triangle_{K, \sigma}\right|} \int_{\triangle_{K, \sigma}} \bar{u}(y) d y-\frac{1}{m(\sigma)} \int_{\sigma} \bar{u}(\xi) d m(\xi)\right|
\end{aligned}
$$

Since $B\left(x_{K}, \alpha h_{K}\right)$ and $\triangle_{K, \sigma}$ are both contained in $K$ which is convex and has diameter $h_{K}$, Lemma 7.1 in the Appendix allows to write

$$
\begin{aligned}
& \left|\frac{1}{\left|B\left(x_{K}, \alpha h_{K}\right)\right|} \int_{B\left(x_{K}, \alpha h_{K}\right)} \bar{u}(x) d x-\frac{1}{\left|\triangle_{K, \sigma}\right|} \int_{\triangle_{K, \sigma}} \bar{u}(y) d y\right|^{2} \\
& \quad \leqslant \frac{C_{1} h_{K}^{N+2}}{\left|B\left(x_{K}, \alpha h_{K}\right)\right|\left|\triangle_{K, \sigma}\right|} \int_{\operatorname{co}\left(B\left(x_{K}, \alpha h_{K}\right) \cup \triangle_{K, \sigma}\right)}|\nabla \bar{u}(z)|^{2} d z \\
& \quad \leqslant \frac{C_{2} h_{K}^{2}}{\left|\triangle_{K, \sigma}\right|} \int_{K}|\nabla \bar{u}(z)|^{2} d z
\end{aligned}
$$

with $C_{1}$ and $C_{2}$ only depending on $(N, \alpha)$. Using this inequality and Lemma 7.2 (from the Appendix) in (3.1), we obtain

$$
\begin{aligned}
\mid \frac{1}{\left|B\left(x_{K}, \alpha h_{K}\right)\right|} \int_{B\left(x_{K}, \alpha h_{K}\right)} \bar{u}(x) d x & -\left.\frac{1}{m(\sigma)} \int_{\sigma} \bar{u}(\xi) d m(\xi)\right|^{2} \\
& \leqslant \frac{C_{3} h_{K}^{2}}{\left|\triangle_{K, \sigma}\right|} \int_{K}|\nabla \bar{u}(z)|^{2} d z
\end{aligned}
$$

where $C_{3}$ only depends on $(N, \alpha)$.

Let $\sigma \in \mathscr{E}_{\text {ext }}$. Since $\bar{u} \in H_{0}^{1}(\Omega)$, (3.2) shows that, denoting by $K$ the cell such that $\sigma \in \mathscr{E}_{K},\left(D_{\sigma} \bar{u}_{\mathscr{T}}\right)^{2} \leqslant \frac{C_{3} h_{K}^{2}}{\left|\triangle_{K, \sigma}\right|} \int_{K}|\nabla \bar{u}(x)|^{2} d x$. We have $\left|\triangle_{K, \sigma}\right|=m(\sigma) d_{\sigma} / N$ (recall that $d_{K, \sigma}=d_{\sigma}$ since $\sigma \in \mathscr{E}_{\text {ext }}$ ); moreover, by Hypothesis (1.3), $\alpha h_{K} \leqslant d_{K, \sigma}=d_{\sigma}$; thus,

$$
\frac{m(\sigma)}{d_{\sigma}}\left(D_{\sigma} \bar{u}_{\mathscr{T}}\right)^{2} \leqslant \frac{N C_{3} h_{K}^{2}}{d_{\sigma}^{2}} \int_{K}|\nabla \bar{u}(x)|^{2} d x \leqslant \frac{N C_{3}}{\alpha^{2}} \int_{K}|\nabla \bar{u}(x)|^{2} d x .
$$


Let $\sigma=K \mid L \in \mathscr{E}_{\text {int }}$. We have

$$
\begin{aligned}
D_{\sigma} \bar{u}_{\mathscr{T}} & \leqslant\left|\frac{1}{\left|B\left(x_{K}, \alpha h_{K}\right)\right|} \int_{B\left(x_{K}, \alpha h_{K}\right)} \bar{u}(x) d x-\frac{1}{m(\sigma)} \int_{\sigma} \bar{u}(\xi) d m(\xi)\right| \\
& +\left|\frac{1}{m(\sigma)} \int_{\sigma} \bar{u}(\xi) d m(\xi)-\frac{1}{\left|B\left(x_{L}, \alpha h_{L}\right)\right|} \int_{B\left(x_{L}, \alpha h_{L}\right)} \bar{u}(x) d x\right|
\end{aligned}
$$

and (3.2) gives thus

$$
\left(D_{\sigma} \bar{u}_{\mathscr{T}}\right)^{2} \leqslant \frac{2 C_{3} h_{K}^{2}}{\left|\triangle_{K, \sigma}\right|} \int_{K}|\nabla \bar{u}(x)|^{2} d x+\frac{2 C_{3} h_{L}^{2}}{\left|\triangle_{L, \sigma}\right|} \int_{L}|\nabla \bar{u}(x)|^{2} d x .
$$

We have $\left|\triangle_{K, \sigma}\right|=m(\sigma) d_{K, \sigma} / N$ and $d_{\sigma} \geqslant d_{K, \sigma} \geqslant \alpha h_{K}$ (and the same properties with $K$ replaced by $L$ ), so that

$$
\frac{m(\sigma)}{d_{\sigma}}\left(D_{\sigma} \bar{u}_{\mathscr{T}}\right)^{2} \leqslant \frac{2 C_{3} N}{\alpha^{2}}\left(\int_{K}|\nabla \bar{u}(x)|^{2} d x+\int_{L}|\nabla \bar{u}(x)|^{2} d x\right)
$$

(3.3) and (3.4) show that, for all $\sigma \in \mathscr{E}$,

$$
\frac{m(\sigma)}{d_{\sigma}}\left(D_{\sigma} \bar{u}_{\mathscr{T}}\right)^{2} \leqslant C_{4} \sum_{K \in \mathscr{T} \mid \sigma \in \mathscr{E}_{K}} \int_{K}|\nabla \bar{u}(x)|^{2} d x
$$

with $C_{4}$ only depending on $(N, \alpha)$. Summing these inequalities on $\sigma \in \mathscr{E}$, we find

$$
\left\|\bar{u}_{\mathscr{T}}\right\|_{1, \mathscr{T}}^{2} \leqslant C_{4} \sum_{\sigma \in \mathscr{E}} \sum_{K \in \mathscr{T} \mid \sigma \in \mathscr{E}_{K}} \int_{K}|\nabla \bar{u}(x)|^{2} d x \leqslant C_{4} \sum_{K \in \mathscr{T}} \int_{K}|\nabla \bar{u}(x)|^{2} d x \operatorname{card}\left(\mathscr{E}_{K}\right)
$$

and (1.4) concludes the proof of the proposition.

Corollary 3.1. Assume that $\mathscr{T}$ is an admissible mesh which satisfies (1.2), (1.3) and (1.4). There exists $C$ only depending on $\left(\Omega,\|\mathbf{v}\|_{\left(L^{\infty}(\Omega)\right)^{N}}, \zeta, \alpha, M\right)$ such that, if $\bar{u}$ is the variational solution to (1.1), $\bar{u}_{\mathscr{T}}$ is defined by (2.1) and $u_{\mathscr{T}}=\left(u_{K}\right)_{K \in \mathscr{T}}$ is the solution to (1.9)—(1.10), then

$$
\left\|\bar{u}_{\mathscr{T}}-u_{\mathscr{T}}\right\|_{1, \mathscr{T}} \leqslant C\left(\|\bar{u}\|_{H_{0}^{1}(\Omega)}+\|f\|_{L^{2}(\Omega)}+\||| G \mid\|_{L^{2}(\Omega)}\right) .
$$

Remark 3.2. By Theorem 2.1 in $[6],\|\bar{u}\|_{H_{0}^{1}(\Omega)}$ is controlled by $\|f\|_{L^{2}(\Omega)}+$ $\||G|\|_{L^{2}(\Omega)}$ and we could thus drop it in the preceding inequality. 
Proof of Corollary 3.1. The right-hand side of (1.9) is written

$$
|K| f_{K}+\sum_{\sigma \in \mathscr{E}_{K}} m(\sigma) Q_{K, \sigma}
$$

with $\left(Q_{K, \sigma}\right)_{K \in \mathscr{T}, \sigma \in \mathscr{E}_{K}}$ which satisfies, for all $\sigma=K \mid L \in \mathscr{E}_{\text {int }}, Q_{K, \sigma}=-Q_{L, \sigma}$ (conservativity); thus, by Proposition 3.2, p.72, in [7], there exists $C_{1}$ only depending on $\left(\Omega,\|\mathbf{v}\|_{\left(L^{\infty}(\Omega)\right)^{N}}, \zeta\right)$ such that

$$
\left\|u_{\mathscr{T}}\right\|_{1, \mathscr{T}} \leqslant C_{1}\left(\sum_{K \in \mathscr{T}}|K| f_{K}^{2}\right)^{1 / 2}+C_{1}\left(\sum_{\sigma \in \mathscr{E}} m(\sigma) d_{\sigma} Q_{\sigma}^{2}\right)^{1 / 2}
$$

where $Q_{\sigma}=\left|Q_{K, \sigma}\right|$ for some $K \in \mathscr{T}$ such that $\sigma \in \mathscr{E}_{K}$ (by conservativity, this definition of $Q_{\sigma}$ does not depend on the choice of such a $K$ ).

Since $\left|\triangle_{K, \sigma}\right|=m(\sigma) d_{K, \sigma} / N$ for all $K \in \mathscr{T}$ and $\sigma \in \mathscr{E}_{K}$, we have, by convexity of $X \rightarrow X^{2}$ and for all $\sigma=K \mid L \in \mathscr{E}_{\text {int }}$,

$$
\begin{aligned}
Q_{\sigma}^{2} & \leqslant \frac{d_{K, \sigma}}{d_{\sigma}} \frac{1}{\left|\triangle_{K, \sigma}\right|} \int_{\triangle_{K, \sigma}}|G(x)|^{2} d x+\frac{d_{L, \sigma}}{d_{\sigma}} \frac{1}{\left|\triangle_{L, \sigma}\right|} \int_{\triangle_{L, \sigma}}|G(x)|^{2} d x \\
& \leqslant \frac{N}{m(\sigma) d_{\sigma}}\left(\int_{\triangle_{K, \sigma}}|G(x)|^{2} d x+\int_{\triangle_{L, \sigma}}|G(x)|^{2} d x\right)
\end{aligned}
$$

(notice that, suppressing the term involving $L$, this estimate is still true if $\sigma \in \mathscr{E}_{K} \cap$ $\left.\mathscr{E}_{\text {ext }}\right)$. Thus,

$$
\begin{aligned}
\sum_{\sigma \in \mathscr{E}} m(\sigma) d_{\sigma} Q_{\sigma}^{2} & \leqslant N \sum_{\sigma \in \mathscr{E}} \sum_{K \in \mathscr{T} \mid \sigma \in \mathscr{E}_{K}} \int_{\triangle_{K, \sigma}}|G(x)|^{2} d x \\
& \leqslant N \sum_{K \in \mathscr{T}} \sum_{\sigma \in \mathscr{E}_{K}} \int_{\triangle_{K, \sigma}}|G(x)|^{2} d x \\
& =N \sum_{K \in \mathscr{T}} \int_{K}|G(x)|^{2} d x=N \int_{\Omega}|G(x)|^{2} d x .
\end{aligned}
$$

Moreover, $f_{K}^{2} \leqslant \frac{1}{|K|} \int_{K}|f(x)|^{2} d x$ and (3.5) gives then

$$
\left\|u_{\mathscr{T}}\right\|_{1, \mathscr{T}} \leqslant C_{1}\|f\|_{L^{2}(\Omega)}+C_{1} \sqrt{N}|||G| \|_{L^{2}(\Omega)} .
$$

Combined with Proposition 3.1, this concludes the proof.

\section{THE $H^{2}$ FRAMEWORK}

Proposition 4.1. Assume that $\mathscr{T}$ is an admissible mesh which satisfies Hypothesis (1.3) and that $\bar{u} \in H^{2}(\Omega) \cap H_{0}^{1}(\Omega)$. Define, for $\sigma=K \mid L \in \mathscr{E}_{\text {int }}$,

$$
R_{K, \sigma}=\frac{\bar{u}_{K}-\bar{u}_{L}}{d_{\sigma}}+\frac{1}{m(\sigma)} \int_{\sigma} \nabla \bar{u}(\xi) \cdot \mathbf{n}_{K, \sigma} d m(\xi) .
$$


and, for $\sigma \in \mathscr{E}_{\text {ext }} \cap \mathscr{E}_{K}$,

$$
R_{K, \sigma}=\frac{\bar{u}_{K}}{d_{\sigma}}+\frac{1}{m(\sigma)} \int_{\sigma} \nabla \bar{u}(\xi) \cdot \mathbf{n}_{K, \sigma} d m(\xi) .
$$

Then there exists $C$ only depending on $(N, \alpha)$ such that

$$
m(\sigma) d_{\sigma} R_{K, \sigma}^{2} \leqslant C \sum_{L \in \mathscr{T} \mid \sigma \in \mathscr{E}_{L}} h_{L}^{2} \int_{L}\left|D^{2} \bar{u}(x)\right|^{2} d x .
$$

Remark 4.1. Notice that this result is false in general if we replace $\bar{u}_{K}$ by the mean value of $\bar{u}$ on $K$ and if $x_{K}$ is not the gravity center of $K$ (consider $\left.K=\right] 0, h{ }^{2}$, $L=]-h, 0[\times] 0, h\left[, x_{K}=(2 h / 3, h / 2), x_{L}=(-2 h / 3, h / 2)\right.$ and $\bar{u}(x, y)=x$; we have then $R_{K, \sigma}=-1 / 4$ but $D^{2} \bar{u}=0$ ).

Remark 4.2. It would be tempting to try to use the Bramble-Hilbert result (see [4] (Theorem 2)) to obtain the estimate of Proposition 4.1 (and also in Proposition 3.1 and Lemmas 7.1, 7.2). This theorem can be used in Finite Element methods thanks to a "reference finite element": for example, in triangular meshes, each finite element can be transformed, by some simple linear application, into some reference triangle; this allows to easily obtain estimates only depending on the size of the element, not its geometry (because they all have the same geometry: that of a triangle).

We do not have such reference control volume in our meshes (our control volumes can have very different geometries); so, in order to prove that the estimates on $R_{K, \sigma}$ only depend on the size of $K$ and not on its geometry, Bramble-Hilbert's result is useless and we have to make the whole proof.

Proof of Proposition 4.1. Due to technical reasons, we must first replace the mean value of $\bar{u}$ on $B\left(x_{K}, \alpha h_{K}\right)$ by the mean value on $B\left(x_{K}, \alpha h_{K} / 2\right)$; step 1 is the study of the consistency error for $\sigma \in \mathscr{E}_{\text {int }}$ with these new mean values (and if $\bar{u}$ is regular). In step 2 , we prove that the error introduced by the use of the mean values on $B\left(x_{K}, \frac{\alpha h_{K}}{2}\right)$ can be controlled, and we conclude the proof for interior edges. In step 3, the case of boundary edges is handled thanks to a symmetry trick which brings us back to the case of interior edges.

Step 1: we suppose that $\bar{u}$ is regular and we take $\sigma=K \mid L \in \mathscr{E}$ int .

We define

$$
\begin{aligned}
R_{K, \sigma}^{\prime}= & \frac{1}{d_{\sigma}}\left(\frac{1}{\left|B\left(x_{K}, \frac{\alpha h_{K}}{2}\right)\right|} \int_{B\left(x_{K}, \frac{\alpha h_{K}}{2}\right)} \bar{u}(x) d x-\frac{1}{\left|B\left(x_{L}, \frac{\alpha h_{L}}{2}\right)\right|} \int_{B\left(x_{L}, \frac{\alpha h_{L}}{2}\right)} \bar{u}(y) d y\right) \\
& +\frac{1}{m(\sigma)} \int_{\sigma} \nabla \bar{u}(\xi) \cdot \mathbf{n}_{K, \sigma} d m(\xi) .
\end{aligned}
$$


Let $\xi \in \sigma$. By Taylor's expansions, we have, for all $x \in B\left(x_{K}, \alpha h_{K} / 2\right)$ and all $y \in B\left(x_{L}, \alpha h_{L} / 2\right)$,

$$
\begin{aligned}
& \bar{u}(x)=\bar{u}(\xi)+\nabla \bar{u}(\xi) \cdot(x-\xi)+\int_{0}^{1}(1-t) D^{2} \bar{u}(\xi+t(x-\xi))(x-\xi) \cdot(x-\xi) d t \\
& \bar{u}(y)=\bar{u}(\xi)+\nabla \bar{u}(\xi) \cdot(y-\xi)+\int_{0}^{1}(1-t) D^{2} \bar{u}(\xi+t(y-\xi))(y-\xi) \cdot(y-\xi) d t .
\end{aligned}
$$

Subtracting these equations and taking the mean value on $\xi \in \sigma$, we find

$$
\begin{aligned}
\bar{u}(x)-\bar{u}(y)= & \frac{1}{m(\sigma)} \int_{\sigma} \nabla \bar{u}(\xi) d m(\xi) \cdot(x-y) \\
& +\frac{1}{m(\sigma)} \int_{\sigma} \int_{0}^{1}(1-t) D^{2} \bar{u}(\xi+t(x-\xi))(x-\xi) \cdot(x-\xi) d t d m(\xi) \\
& -\frac{1}{m(\sigma)} \int_{\sigma} \int_{0}^{1}(1-t) D^{2} \bar{u}(\xi+t(y-\xi))(y-\xi) \cdot(y-\xi) d t d m(\xi) .
\end{aligned}
$$

We now take the mean values on $x \in B\left(x_{K}, \alpha h_{K} / 2\right)$ and $y \in B\left(x_{L}, \alpha h_{L} / 2\right)$; since the mean values of $x \rightarrow x$ and $y \rightarrow y$ on these sets are respectively $x_{K}$ and $x_{L}$ and since $x_{L}-x_{K}=d_{\sigma} \mathbf{n}_{K, \sigma}$, dividing by $d_{\sigma}$, we obtain

$$
\begin{aligned}
R_{K, \sigma}^{\prime}= & \frac{1}{d_{\sigma} m(\sigma)\left|B\left(x_{K}, \frac{\alpha h_{K}}{2}\right)\right|} \int_{B\left(x_{K}, \frac{\alpha h_{K}}{2}\right)} \int_{\sigma} \int_{0}^{1} F(t, x, \xi) d t d m(\xi) d x \\
& -\frac{1}{d_{\sigma} m(\sigma)\left|B\left(x_{L}, \frac{\alpha h_{L}}{2}\right)\right|} \int_{B\left(x_{L}, \frac{\alpha h_{L}}{2}\right)} \int_{\sigma} \int_{0}^{1} F(t, y, \xi) d t d m(\xi) d y
\end{aligned}
$$

with $F(t, x, \xi)=(1-t) D^{2} \bar{u}(\xi+t(x-\xi))(x-\xi) \cdot(x-\xi)$.

For $x \in K$ and $\xi \in \sigma$, we have $|x-\xi| \leqslant h_{K}$, so that $|F(t, x, \xi)| \leqslant h_{K}^{2}(1-$ $t)\left|D^{2} u(\xi+t(x-\xi))\right| ;$ Jensen's inequality gives then

$$
\begin{aligned}
\left(R_{K, \sigma}^{\prime}\right)^{2} \leqslant & \frac{2 h_{K}^{4}}{d_{\sigma}^{2} m(\sigma)\left|B\left(x_{K}, \frac{\alpha h_{K}}{2}\right)\right|} \times \\
& \int_{B\left(x_{K}, \frac{\alpha h_{K}}{2}\right)} \int_{\sigma} \int_{0}^{1}(1-t)^{2}\left|D^{2} \bar{u}(\xi+t(x-\xi))\right|^{2} d t d m(\xi) d x \\
+ & \frac{2 h_{L}^{4}}{d_{\sigma}^{2} m(\sigma)\left|B\left(x_{L}, \frac{\alpha h_{L}}{2}\right)\right|} \times \\
& \int_{B\left(x_{L}, \frac{\alpha h_{L}}{2}\right)} \int_{\sigma} \int_{0}^{1}(1-t)^{2}\left|D^{2} \bar{u}(\xi+t(y-\xi))\right|^{2} d t d m(\xi) d y .
\end{aligned}
$$

By translation, we can suppose that $\sigma=\{0\} \times \widetilde{\sigma} \subset\{0\} \times \mathbb{R}^{N-1}$. Let $x \in$ $B\left(x_{K}, \alpha h_{K} / 2\right)$; we use the change of variable $\left.\left(t, \xi^{\prime}\right) \in\right] 0,1\left[\times \widetilde{\sigma} \rightarrow z=\left(0, \xi^{\prime}\right)+t(x-\right.$ 
$\left.\left(0, \xi^{\prime}\right)\right) \in V_{x} \subset K\left(K\right.$ is convex), whose jacobian determinant is $\left|x_{1}\right|(1-t)^{N-1}$ (where $x_{1}$ is the first component of $x$ - we will see below that $x_{1} \neq 0$ ). Since $N \leqslant 3$, we have, if $t \in] 0,1\left[,(1-t)^{2} \leqslant(1-t)^{N-1}\right.$ and we can thus write

$$
\begin{aligned}
& \int_{\sigma} \int_{0}^{1}(1-t)^{2}\left|D^{2} \bar{u}(\xi+t(x-\xi))\right|^{2} d t d m(\xi) \\
& \quad \leqslant \int_{\sigma} \int_{0}^{1}(1-t)^{N-1}\left|D^{2} \bar{u}(\xi+t(x-\xi))\right|^{2} d t d m(\xi) \\
& \quad \leqslant\left|x_{1}\right|^{-1} \int_{K}\left|D^{2} \bar{u}(z)\right|^{2} d z .
\end{aligned}
$$

Write $x_{K}=(a, b)$ with $a \in \mathbb{R}$ and $b \in \mathbb{R}^{N-1}$. The straight line going through $x_{K}$ and orthogonal to $\sigma \subset\{0\} \times \mathbb{R}^{N-1}$, (i.e. the line $\mathbb{R} \times\{b\}$ ) intersects $\sigma$ (i.e. $(0, b) \in \sigma)$. Thus, $|a|=|(a, b)-(0, b)| \geqslant \operatorname{dist}\left(x_{K}, \sigma\right) \geqslant \operatorname{dist}\left(x_{K}, \partial K\right) \geqslant \alpha h_{K}$ (recall that $\left.B\left(x_{K}, \alpha h_{K}\right) \in K\right)$. Thus, if $x \in B\left(x_{K}, \alpha h_{K} / 2\right)$, we have $\left|x_{1}\right| \geqslant|a|-\left|x_{1}-a\right| \geqslant$ $\alpha h_{K}-\left|x-x_{K}\right| \geqslant \frac{\alpha h_{K}}{2}$ and (4.2) gives then

$$
\int_{\sigma} \int_{0}^{1}(1-t)^{2}\left|D^{2} \bar{u}(\xi+t(x-\xi))\right|^{2} d t d m(\xi) \leqslant \frac{2}{\alpha h_{K}} \int_{K}\left|D^{2} \bar{u}(z)\right|^{2} d z .
$$

Therefore,

$$
\begin{gathered}
\frac{1}{\left|B\left(x_{K}, \frac{\alpha h_{K}}{2}\right)\right|} \int_{B\left(x_{K}, \frac{\alpha h_{K}}{2}\right)} \int_{\sigma} \int_{0}^{1}(1-t)^{2}\left|D^{2} \bar{u}(\xi+t(x-\xi))\right|^{2} d t d m(\xi) d x \\
\leqslant \frac{2}{\alpha h_{K}} \int_{K}\left|D^{2} \bar{u}(z)\right|^{2} d z
\end{gathered}
$$

Coming back to (4.1) (and using the preceding inequality also with $L$ instead of $K)$, we obtain

$$
\left(R_{K, \sigma}^{\prime}\right)^{2} \leqslant \frac{4 h_{K}^{3}}{\alpha d_{\sigma}^{2} m(\sigma)} \int_{K}\left|D^{2} \bar{u}(z)\right|^{2} d z+\frac{4 h_{L}^{3}}{\alpha d_{\sigma}^{2} m(\sigma)} \int_{L}\left|D^{2} \bar{u}(z)\right|^{2} d z
$$

Since $d_{\sigma} \geqslant d_{K, \sigma} \geqslant \alpha h_{K}$, we deduce that

$$
m(\sigma) d_{\sigma}\left(R_{K, \sigma}^{\prime}\right)^{2} \leqslant \frac{4 h_{K}^{2}}{\alpha^{2}} \int_{K}\left|D^{2} \bar{u}(z)\right|^{2} d z+\frac{4 h_{L}^{2}}{\alpha^{2}} \int_{L}\left|D^{2} \bar{u}(z)\right|^{2} d z
$$

Step 2: we now estimate the difference between the mean values of $\bar{u}$ on $B\left(x_{K}, \alpha h_{K}\right)$ and on $B\left(x_{K}, \frac{\alpha h_{K}}{2}\right)$, and we conclude for $\sigma \in \mathscr{E}_{\text {int }}$.

Let $v(x)=\bar{u}(x)-\left(\frac{1}{\left|B\left(x_{K}, \alpha h_{K}\right)\right|} \int_{B\left(x_{K}, \alpha h_{K}\right)} \nabla \bar{u}(y) d y\right) \cdot\left(x-x_{K}\right)$. We have, for $x \in$ $B\left(0, \alpha h_{K}\right)$,

$$
v\left(x_{K}+x\right)-v\left(x_{K}+\frac{x}{2}\right)=\int_{0}^{1} \nabla v\left(x_{K}+\frac{1+t}{2} x\right) \cdot \frac{x}{2} d t .
$$


Integrating on $x \in B\left(0, \alpha h_{K}\right)$, dividing by $\left|B\left(x_{K}, \alpha h_{K}\right)\right|$ and thanks to the change of variable $y=x_{K}+\frac{x}{2}$ in the second integral, we find

$$
\begin{gathered}
\frac{1}{\left|B\left(x_{K}, \alpha h_{K}\right)\right|} \int_{B\left(x_{K}, \alpha h_{K}\right)} v(x) d x-\frac{1}{\left|B\left(x_{K}, \frac{\alpha h_{K}}{2}\right)\right|} \int_{B\left(x_{K}, \frac{\alpha h_{K}}{2}\right)} v(y) d y \\
=\frac{1}{\left|B\left(x_{K}, \alpha h_{K}\right)\right|} \int_{B\left(0, \alpha h_{K}\right)} \int_{0}^{1} \nabla v\left(x_{K}+\frac{1+t}{2} x\right) \cdot \frac{x}{2} d t d x .
\end{gathered}
$$

Since the mean values on $B\left(x_{K}, \alpha h_{K}\right)$ and on $B\left(x_{K}, \frac{\alpha h_{K}}{2}\right)$ of $x \rightarrow x-x_{K}$ are null, the mean values of $v$ on these sets are equal to the mean values of $\bar{u}$ on the same sets. Thus, denoting

$$
I_{K, \sigma}=\frac{1}{d_{\sigma}}\left(\frac{1}{\left|B\left(x_{K}, \alpha h_{K}\right)\right|} \int_{B\left(x_{K}, \alpha h_{K}\right)} \bar{u}(x) d x-\frac{1}{\left|B\left(x_{K}, \frac{\alpha h_{K}}{2}\right)\right|} \int_{B\left(x_{K}, \frac{\alpha h_{K}}{2}\right)} \bar{u}(y) d y\right),
$$

we have just proved that

$$
\left|I_{K, \sigma}\right| \leqslant \frac{\alpha h_{K}}{2 d_{\sigma}\left|B\left(x_{K}, \alpha h_{K}\right)\right|} \int_{B\left(0, \alpha h_{K}\right)} \int_{0}^{1}\left|\nabla v\left(x_{K}+\frac{1+t}{2} x\right)\right| d t d x .
$$

Using the change of variable $x \rightarrow z=x_{K}+\frac{1+t}{2} x$ (which sends $B\left(0, \alpha h_{K}\right)$ into (but not onto) $B\left(x_{K}, \alpha h_{K}\right)$ ), we deduce

$$
\begin{aligned}
\left(I_{K, \sigma}\right)^{2} & \leqslant \frac{\alpha^{2} h_{K}^{2}}{4 d_{\sigma}^{2}}\left(\frac{1}{\left|B\left(x_{K}, \alpha h_{K}\right)\right|} \int_{B\left(x_{K}, \alpha h_{K}\right)} \int_{0}^{1}|\nabla v(z)|\left(\frac{2}{1+t}\right)^{N} d t d z\right)^{2} \\
& \leqslant \frac{2^{2 N-2} \alpha^{2} h_{K}^{2}}{d_{\sigma}^{2}}\left(\frac{1}{\left|B\left(x_{K}, \alpha h_{K}\right)\right|} \int_{B\left(x_{K}, \alpha h_{K}\right)}|\nabla v(z)| d z\right)^{2}
\end{aligned}
$$

We have $\nabla v(z)=\nabla \bar{u}(z)-\frac{1}{\left|B\left(x_{K}, \alpha h_{K}\right)\right|} \int_{B\left(x_{K}, \alpha h_{K}\right)} \nabla \bar{u}(y) d y$. Thus, thanks to Lemma 7.1,

$$
\begin{aligned}
& \left(\frac{1}{\left|B\left(x_{K}, \alpha h_{K}\right)\right|} \int_{B\left(x_{K}, \alpha h_{K}\right)}|\nabla v(z)| d z\right)^{2} \\
& \quad \leqslant\left(\frac{1}{\left|B\left(x_{K}, \alpha h_{K}\right)\right|\left|B\left(x_{K}, \alpha h_{K}\right)\right|} \int_{B\left(x_{K}, \alpha h_{K}\right)} \int_{B\left(x_{K}, \alpha h_{K}\right)}|\nabla \bar{u}(z)-\nabla \bar{u}(y)| d z d y\right)^{2} \\
& \quad \leqslant \frac{C_{1} h_{K}^{N+2}}{\left|B\left(x_{K}, \alpha h_{K}\right)\right|\left|B\left(x_{K}, \alpha h_{K}\right)\right|} \int_{B\left(x_{K}, \alpha h_{K}\right)}\left|D^{2} \bar{u}(x)\right|^{2} d x \\
& \quad \leqslant \frac{C_{2} h_{K}^{2}}{\left|B\left(x_{K}, \alpha h_{K}\right)\right|} \int_{B\left(x_{K}, \alpha h_{K}\right)}\left|D^{2} \bar{u}(x)\right|^{2} d x
\end{aligned}
$$

where $C_{1}$ and $C_{2}$ only depend on $(N, \alpha)$. 
Coming back to (4.4), we obtain $C_{3}$ only depending on $(N, \alpha)$ such that

$$
\left(I_{K, \sigma}\right)^{2} \leqslant \frac{C_{3} h_{K}^{4}}{d_{\sigma}^{2}\left|B\left(x_{K}, \alpha h_{K}\right)\right|} \int_{B\left(x_{K}, \alpha h_{K}\right)}\left|D^{2} \bar{u}(x)\right|^{2} d x .
$$

$\sigma$ is of diameter less than $h_{K}$, so that $m(\sigma) \leqslant C_{4} h_{K}^{N-1}$ with $C_{4}$ only depending on $N$. Moreover, $d_{\sigma} \geqslant d_{K, \sigma} \geqslant \alpha h_{K}$; hence,

$$
\begin{aligned}
m(\sigma) d_{\sigma}\left(I_{K, \sigma}\right)^{2} & \leqslant \frac{C_{5} h_{K}^{N+3}}{\alpha h_{K}\left|B\left(x_{K}, \alpha h_{K}\right)\right|} \int_{B\left(x_{K}, \alpha h_{K}\right)}\left|D^{2} \bar{u}(x)\right|^{2} d x \\
& \leqslant C_{6} h_{K}^{2} \int_{K}\left|D^{2} \bar{u}(x)\right|^{2} d x
\end{aligned}
$$

with $C_{5}$ and $C_{6}$ only depending on $(N, \alpha)$.

We have $R_{K, \sigma}=I_{K, \sigma}+R_{K, \sigma}^{\prime}-I_{L, \sigma}$. Thanks to (4.3) and (4.5), we deduce

$$
m(\sigma) d_{\sigma} R_{K, \sigma}^{2} \leqslant C_{7} h_{K}^{2} \int_{K}\left|D^{2} \bar{u}(x)\right|^{2} d x+C_{7} h_{L}^{2} \int_{L}\left|D^{2} \bar{u}(x)\right|^{2} d x
$$

with $C_{7}$ only depending on $(N, \alpha)$. This estimate has been obtained for $\bar{u}$ regular, but, by the density result of Lemma 7.3 (found in the Appendix), it is also satisfied by functions in $H^{2}(K \cup \sigma \cup L)$ (thus by functions in $\left.H^{2}(\Omega)\right)$. This concludes the proof if $\sigma \in \mathscr{E}_{\text {int }}$.

Step 3: suppose now that $\sigma \in \mathscr{E}_{\mathrm{ext}} \cap \mathscr{E}_{K}$.

Since $\bar{u} \in H^{2}(\Omega) \cap H_{0}^{1}(\Omega)$, we have $\bar{u}=0$ on $\sigma$. Denoting by $S$ the orthogonal symmetry with respect to the hyperplane generated by $\sigma$, it is then well known that the function $\mathscr{U}: K \cup \sigma \cup S(K) \rightarrow \mathbb{R}$ which is equal to $\bar{u}$ on $K$ and to $-\bar{u} \circ S$ on $S(K)$ belongs to $H^{2}(K \cup \sigma \cup S(K))$.

We notice that all the hypotheses on $\left(K, x_{K}, \sigma, L, x_{L}\right)$ used in Steps 1 and 2 (and in the proof of Lemma 7.3) are satisfied here by $\left(K, x_{K}, \sigma, S(K), S\left(x_{K}\right)\right.$ ) (with $\operatorname{dist}\left(x_{K}, S\left(x_{K}\right)\right)=2 \operatorname{dist}\left(x_{K}, \sigma\right)=2 d_{\sigma}$ instead of $\left.d_{\sigma}\right)$.

The result (4.6) of Step 2 hence applies with $\mathscr{U}$ instead of $\bar{u}$ and we can write, defining $\mathscr{U}_{K}$ and $\mathscr{U}_{S(K)}$ as the mean values of $\mathscr{U}$ on $B\left(x_{K}, \alpha h_{K}\right)$ and $B\left(x_{S(K)}, \alpha h_{S(K)}\right)$ respectively,

$$
\begin{gathered}
2 m(\sigma) d_{\sigma}\left(\frac{\mathscr{U}_{K}-\mathscr{U}_{S(K)}}{2 d_{\sigma}}+\frac{1}{m(\sigma)} \int_{\sigma} \nabla \mathscr{U}(\xi) \cdot \mathbf{n}_{K, \sigma} d m(\xi)\right)^{2} \\
\leqslant C_{7} h_{K}^{2} \int_{K}\left|D^{2} \mathscr{U}(x)\right|^{2} d x+C_{7} h_{S(K)}^{2} \int_{S(K)}\left|D^{2} \mathscr{U}(x)\right|^{2} d x .
\end{gathered}
$$

Since $\mathscr{U}=\bar{u}$ on $K$, we have $\mathscr{U}_{K}=\bar{u}_{K}$ and, in the sense of the traces, $\nabla \mathscr{U}=\nabla \bar{u}$ on $\sigma$. Moreover, $\mathscr{U}=-\bar{u} \circ S$ on $S(K)$, so that $\mathscr{U}_{S(K)}=-\bar{u}_{K}$ (notice that $h_{S(K)}=h_{K}$, 
which implies $\left.B\left(S\left(x_{K}\right), \alpha h_{S(K)}\right)=S\left(B\left(x_{K}, \alpha h_{K}\right)\right)\right)$ and $\left|D^{2} \mathscr{U}(x)\right|=\left|D^{2} \bar{u}(S(x))\right|$ for $x \in S(K)$. Thus, the preceding estimate yields

$$
2 m(\sigma) d_{\sigma}\left(\frac{\bar{u}_{K}}{d_{\sigma}}+\frac{1}{m(\sigma)} \int_{\sigma} \nabla \bar{u}(\xi) \cdot \mathbf{n}_{K, \sigma} d m(\xi)\right)^{2} \leqslant 2 C_{7} h_{K}^{2} \int_{K}\left|D^{2} \bar{u}(x)\right|^{2} d x,
$$

which is exactly the desired result for $\sigma \in \mathscr{E}_{\text {ext }}$.

Theorem 4.1. Assume that $G \in\left(H^{1}(\Omega)\right)^{N}$ and that the variational solution $\bar{u}$ to (1.1) belongs to $H^{2}(\Omega) \cap H_{0}^{1}(\Omega)$. If $\mathscr{T}$ is an admissible mesh which satisfies (1.2), (1.3) and (1.4), there exists $C$ only depending on $\left(\Omega,\|\mathbf{v}\|_{\left(L^{\infty}(\Omega)\right)^{N}}, \| b||_{L^{\infty}(\Omega)}, \zeta, \alpha, M\right)$ such that, $\bar{u}_{\mathscr{T}}$ being defined by (2.1) and $u_{\mathscr{T}}=\left(u_{K}\right)_{K \in \mathscr{T}}$ being the solution to (1.9)(1.10),

$$
\left\|\bar{u}_{\mathscr{T}}-u_{\mathscr{T}}\right\|_{1, \mathscr{T}} \leqslant C\left(\|\bar{u}\|_{H^{2}(\Omega)}+\||\nabla G|\|_{L^{2}(\Omega)}\right) h_{\mathscr{T}} .
$$

Remark 4.3. A similar result, with $\bar{u}_{K}$ replaced by $\bar{u}\left(x_{K}\right)$ and $G=0$, is proved (using (1.3)) in [9] and [10] (Theorem 3.2). Here, we also need (1.4) because, $\bar{u}_{K}$ taking into account all the values of $\bar{u}$ on a ball around $x_{K}$, we cannot control $R_{K, \sigma}$ in Proposition 4.1 (for example) only by means of $\int_{\triangle_{K, \sigma}}\left|D^{2} \bar{u}\right|^{2}$, as it is done in [10].

Proof of Theorem 4.1. By (1.1), $\operatorname{div}(\mathbf{v} \bar{u}) \in L^{2}(\Omega) \subset L^{1}(\Omega)$ and, $\mathbf{v}$ and $\bar{u}$ being continuous on $\bar{\Omega}$ (because $\bar{u} \in H^{2}(\Omega)$ and $N \leqslant 3$ ), we can apply Lemma 7.4 (see the Appendix) to compute the integral of $\operatorname{div}(\mathbf{v} \bar{u})$ on a convex open subset of $\Omega$. Thus, integrating (1.1) on a control volume $K \in \mathscr{T}$, we obtain

$$
\begin{aligned}
& -\sum_{\sigma \in \mathscr{E}_{K}} \int_{\sigma} \nabla \bar{u}(\xi) \cdot \mathbf{n}_{K, \sigma} d m(\xi)+\sum_{\sigma \in \mathscr{E}_{K}} \int_{\sigma} \mathbf{v}(\xi) \cdot \mathbf{n}_{K, \sigma} \bar{u}(\xi) d m(\xi) \\
& \quad+\int_{K} b(x) \bar{u}(x) d x=|K| f_{K}+\sum_{\sigma \in \mathscr{E}_{K}} \int_{\sigma} G(\xi) \cdot \mathbf{n}_{K, \sigma} d m(\xi) .
\end{aligned}
$$

Denote, as in Proposition 4.1,

$$
R_{K, \sigma}=\frac{\bar{u}_{K}-\bar{u}_{L}}{d_{\sigma}}-\left(-\frac{1}{m(\sigma)} \int_{\sigma} \nabla \bar{u}(\xi) \cdot \mathbf{n}_{K, \sigma} d m(\xi)\right)
$$

with $\sigma=K \mid L$ if $\sigma \in \mathscr{E}_{K} \cap \mathscr{E}_{\text {int }}$ and $\bar{u}_{L}=0$ if $\sigma \in \mathscr{E}_{K} \cap \mathscr{E}_{\text {ext }}$.

Let

$$
r_{K, \sigma}=v_{K, \sigma} \bar{u}_{\sigma,+}-\frac{1}{m(\sigma)} \int_{\sigma} \mathbf{v}(\xi) \cdot \mathbf{n}_{K, \sigma} \bar{u}(\xi) d m(\xi)
$$


where $\bar{u}_{\sigma,+}=\bar{u}_{K}$ if $v_{K, \sigma} \geqslant 0, \bar{u}_{\sigma,+}=\bar{u}_{L}$ if $v_{K, \sigma}<0$ and $\sigma=K \mid L \in \mathscr{E}_{K} \cap \mathscr{E}_{\text {int }}$, and $\bar{u}_{\sigma,+}=0$ if $v_{K, \sigma}<0$ and $\sigma \in \mathscr{E}_{K} \cap \mathscr{E}_{\text {ext }}$.

Finally, define

$$
\rho_{K}=b_{K} \bar{u}_{K}-\frac{1}{|K|} \int_{K} b(x) \bar{u}(x) d x
$$

and

$$
M_{K, \sigma}=\frac{1}{m(\sigma)} \int_{\sigma} G(\xi) \cdot \mathbf{n}_{K, \sigma} d m(\xi)-\left(\frac{d_{K, \sigma}}{d_{\sigma}} G_{K, \sigma}-\frac{d_{L, \sigma}}{d_{\sigma}} G_{L, \sigma}\right)
$$

(with the convention that $\sigma=K \mid L$ if $\sigma \in \mathscr{E}_{K} \cap \mathscr{E}_{\text {int }}$ and that $d_{L, \sigma}=G_{L, \sigma}=0$ if $\left.\sigma \in \mathscr{E}_{K} \cap \mathscr{E}_{\text {ext }}\right)$.

(4.7) shows that $\left(\bar{u}_{K}\right)_{K \in \mathscr{T}}$ satisfies (1.9)—(1.10), provided that we add

$$
|K| \rho_{K}+\sum_{\sigma \in \mathscr{E}_{K}} m(\sigma)\left(R_{K, \sigma}+r_{K, \sigma}+M_{K, \sigma}\right)
$$

to the right-hand side of (1.9). Therefore, subtracting the equations satisfied by $\left(u_{K}\right)_{K \in \mathscr{T}}$ to the equations satisfied by $\left(\bar{u}_{K}\right)_{K \in \mathscr{T}}$, we see that $\left(e_{K}\right)_{K \in \mathscr{T}}=\left(\bar{u}_{K}-\right.$ $\left.u_{K}\right)_{K \in \mathscr{T}}$ satisfies

$$
\begin{array}{r}
\forall K \in \mathscr{T}, \sum_{\sigma \in \mathscr{E}_{K}} \frac{m(\sigma)}{d_{\sigma}}\left(e_{K}-e_{L}\right)+\sum_{\sigma \in \mathscr{E}_{K}} m(\sigma) v_{K, \sigma} e_{\sigma,+}+|K| b_{K} e_{K} \\
=|K| \rho_{K}+\sum_{\sigma \in \mathscr{E}_{K}} m(\sigma)\left(R_{K, \sigma}+r_{K, \sigma}+M_{K, \sigma}\right)
\end{array}
$$

(where $\sigma=K \mid L$ if $\sigma \in \mathscr{E}_{K} \cap \mathscr{E}_{\text {int }}$ and $e_{L}=d_{L, \sigma}=G_{L, \sigma}=0$ if $\sigma \in \mathscr{E}_{K} \cap \mathscr{E}_{\text {ext }}$ ),

$$
\begin{array}{lll}
\forall \sigma=K \mid L \in \mathscr{E}_{\text {int }}, & e_{\sigma,+}=e_{K} \text { if } v_{K, \sigma} \geqslant 0, & e_{\sigma,+}=e_{L} \text { otherwise, } \\
\forall \sigma \in \mathscr{E}_{\text {ext }} \cap \mathscr{E}_{K}, & e_{\sigma,+}=e_{K} \text { if } v_{K, \sigma} \geqslant 0, & e_{\sigma,+}=0 \text { otherwise. }
\end{array}
$$

By definition, $\left(R_{K, \sigma}\right)_{K \in \mathscr{T}, \sigma \in \mathscr{E}_{K}},\left(r_{K, \sigma}\right)_{K \in \mathscr{T}, \sigma \in \mathscr{E}_{K}}$ and $\left(M_{K, \sigma}\right)_{K \in \mathscr{T}, \sigma \in \mathscr{E}_{K}}$ are conservative: for all $\sigma=K \mid L \in \mathscr{E}_{\text {int }}$, we have $R_{K, \sigma}=-R_{L, \sigma}, r_{K, \sigma}=-r_{L, \sigma}$ and $M_{K, \sigma}=$ $-M_{L, \sigma}$ (notice that $\mathbf{n}_{K, \sigma}=-\mathbf{n}_{L, \sigma}$ ). Hence, by Proposition 3.2 in [7], we deduce that there exists $C_{0}$ only depending on $\left(\Omega,\|\mathbf{v}\|_{\left(L^{\infty}(\Omega)\right)^{N}}, \zeta\right)$ such that

$$
\left\|e_{\mathscr{T}}\right\|_{1, \mathscr{T}} \leqslant C_{0}\left(\sum_{K \in \mathscr{T}}|K| \rho_{K}^{2}\right)^{1 / 2}+C_{0}\left(\sum_{\sigma \in \mathscr{E}} m(\sigma) d_{\sigma} A_{\sigma}^{2}\right)^{1 / 2},
$$

where we have denoted $A_{\sigma}=\left|R_{K, \sigma}+r_{K, \sigma}+M_{K, \sigma}\right|$ for some $K \in \mathscr{T}$ such that $\sigma \in \mathscr{E}_{K}$ (by conservativity of these quantities, this definition does not depend on the choice of such a $K$ ).

We have

$$
\begin{aligned}
\left|\rho_{K}\right| & =\left|\frac{1}{|K|} \int_{K} b(x)\left(\bar{u}_{K}-\bar{u}(x)\right) d x\right| \\
& \leqslant \frac{\|\left. b\right|_{L^{\infty}(\Omega)}}{|K|\left|B\left(x_{K}, \alpha h_{K}\right)\right|} \int_{K} \int_{B\left(x_{K}, \alpha h_{K}\right)}|\bar{u}(y)-\bar{u}(x)| d y d x .
\end{aligned}
$$


Therefore, by Lemma 7.1, there exists $C_{1}$ only depending on $\left(N,\|b\|_{L^{\infty}(\Omega)}, \alpha\right)$ such that

$$
|K| \rho_{K}^{2} \leqslant C_{1} h_{K}^{2} \int_{K}|\nabla \bar{u}(x)|^{2} d x
$$

By definition,

$$
\begin{aligned}
\left|r_{K, \sigma}\right| & =\left|\frac{1}{m(\sigma)} \int_{\sigma} \mathbf{v}(\xi) \cdot \mathbf{n}_{K, \sigma}\left(\bar{u}_{\sigma,+}-\bar{u}(\xi)\right) d m(\xi)\right| \\
& \leqslant \frac{\||\mathbf{v}|\|_{L^{\infty}(\Omega)}}{m(\sigma)} \int_{\sigma}\left|\bar{u}_{\sigma,+}-\bar{u}(\xi)\right| d m(\xi)
\end{aligned}
$$

and either $\bar{u}_{\sigma,+}=\bar{u}_{L}$ for some $L \in \mathscr{T}$ such that $\sigma \in \mathscr{E}_{L}$, or $\bar{u}_{\sigma,+}=0$ and $\sigma \in \mathscr{E}_{\text {ext }}$. In the second case, since $u \in H_{0}^{1}(\Omega)$, we obtain $\left|r_{K, \sigma}\right| \leqslant 0$ (i.e. $r_{K, \sigma}=0$ ). In the first case, we define $v=\left|\bar{u}_{L}-\bar{u}\right| \in H^{1}(\Omega)$ and, using Lemma 7.2, we see that

$$
\begin{aligned}
( & \left.\frac{1}{m(\sigma)} \int_{\sigma}\left|\bar{u}_{L}-\bar{u}(\xi)\right| d m(\xi)\right)^{2} \\
= & \left(\frac{1}{m(\sigma)} \int_{\sigma} v(\xi) d m(\xi)\right)^{2} \\
\leqslant & 2\left(\frac{1}{m(\sigma)} \int_{\sigma} v(\xi) d m(\xi)-\frac{1}{\left|\triangle_{L, \sigma}\right|} \int_{\triangle_{L, \sigma}} v(x) d x\right)^{2} \\
& +2\left(\frac{1}{\left|\triangle_{L, \sigma}\right|} \int_{\triangle_{L, \sigma}} v(x) d x\right)^{2} \\
\leqslant & \frac{C_{2} h_{L}^{2}}{\left|\triangle_{L, \sigma}\right|} \int_{\triangle_{L, \sigma}}|\nabla v(x)|^{2} d x+2\left(\frac{1}{\left|\triangle_{L, \sigma}\right|} \int_{\triangle_{L, \sigma}} v(x) d x\right)^{2}
\end{aligned}
$$

with $C_{2}$ only depending on $(N, \alpha)$. But $\nabla v=\operatorname{sgn}\left(\bar{u}_{L}-\bar{u}\right) \nabla \bar{u}$ and

$$
\begin{aligned}
v(x) & =\left|\frac{1}{\left|B\left(x_{L}, \alpha h_{L}\right)\right|} \int_{B\left(x_{L}, \alpha h_{L}\right)} \bar{u}(y) d y-\bar{u}(x)\right| \\
& \leqslant \frac{1}{\left|B\left(x_{L}, \alpha h_{L}\right)\right|} \int_{B\left(x_{L}, \alpha h_{L}\right)}|\bar{u}(y)-\bar{u}(x)| d y,
\end{aligned}
$$


so that, by Lemma 7.1,

$$
\begin{aligned}
& \left(\frac{1}{m(\sigma)} \int_{\sigma}\left|\bar{u}_{L}-\bar{u}(\xi)\right| d m(\xi)\right)^{2} \\
& \leqslant \frac{C_{2} h_{L}^{2}}{\left|\triangle_{L, \sigma}\right|} \int_{\triangle_{L, \sigma}}|\nabla \bar{u}(x)|^{2} d x \\
& \quad+2\left(\frac{1}{\left|\triangle_{L, \sigma}\right|\left|B\left(x_{L}, \alpha h_{L}\right)\right|} \int_{\triangle_{L, \sigma}} \int_{B\left(x_{L}, \alpha h_{L}\right)}|\bar{u}(y)-\bar{u}(x)| d y d x\right)^{2} \\
& \quad \leqslant \frac{C_{3} h_{L}^{2}}{\left|\triangle_{L, \sigma}\right|} \int_{L}|\nabla \bar{u}(x)|^{2} d x
\end{aligned}
$$

where $C_{3}$ only depends on $(N, \alpha)$. Using this in (4.10), and since

$$
\left|\triangle_{L, \sigma}\right|=m(\sigma) d_{L, \sigma} / N \geqslant \alpha m(\sigma) h_{L} / N
$$

(because $d_{L, \sigma}=\operatorname{dist}\left(x_{L}, \sigma\right) \geqslant \operatorname{dist}\left(x_{L}, \partial L\right)$ and $\left.B\left(x_{L}, \alpha h_{L}\right) \subset L\right)$, we deduce that there exists $C_{4}$ only depending on $\left(N,\|\mathbf{v}\|_{\left(L^{\infty}(\Omega)\right)^{N}}, \alpha\right)$ such that

$$
\begin{aligned}
m(\sigma) d_{\sigma} r_{K, \sigma}^{2} & \leqslant C_{3} \|\left.\mathbf{v}\right|_{\left(L^{\infty}(\Omega)\right)^{N}} ^{2} \frac{m(\sigma) d_{\sigma} h_{L}^{2}}{\left|\triangle_{L, \sigma}\right|} \int_{L}|\nabla u(x)|^{2} d x \\
& \leqslant C_{4} h_{\mathscr{T}} h_{L} \int_{L}|\nabla u(x)|^{2} d x
\end{aligned}
$$

for some $L \in \mathscr{T}$ such that $\sigma \in \mathscr{E}_{L}$ (we have used the fact that $d_{\sigma} \leqslant 2 h_{\mathscr{T}}$ ). Notice that this estimate is also true (for any $L \in \mathscr{T}$...) in the case where $u_{\sigma,+}=0$ with $\sigma \in \mathscr{E}_{\text {ext }}$, since $r_{K, \sigma}$ is then null.

We have, for $\sigma=K \mid L \in \mathscr{E}_{\text {int }}$, since $d_{K, \sigma}+d_{L, \sigma}=d_{\sigma}$,

$$
\begin{aligned}
M_{K, \sigma}= & \frac{d_{K, \sigma}}{d_{\sigma}}\left(\frac{1}{m(\sigma)} \int_{\sigma} G(\xi) \cdot \mathbf{n}_{K, \sigma} d m(\xi)-\frac{1}{\left|\triangle_{K, \sigma}\right|} \int_{\triangle_{K, \sigma}} G(x) \cdot \mathbf{n}_{K, \sigma} d x\right) \\
& +\frac{d_{L, \sigma}}{d_{\sigma}}\left(\frac{1}{m(\sigma)} \int_{\sigma} G(\xi) \cdot \mathbf{n}_{K, \sigma} d m(\xi)+\frac{1}{\left|\triangle_{L, \sigma}\right|} \int_{\triangle_{L, \sigma}} G(x) \cdot \mathbf{n}_{L, \sigma} d x\right) \\
= & \frac{d_{K, \sigma}}{d_{\sigma}}\left(\frac{1}{m(\sigma)} \int_{\sigma} G(\xi) d m(\xi)-\frac{1}{\left|\triangle_{K, \sigma}\right|} \int_{\triangle_{K, \sigma}} G(x) d x\right) \cdot \mathbf{n}_{K, \sigma} \\
& +\frac{d_{L, \sigma}}{d_{\sigma}}\left(\frac{1}{m(\sigma)} \int_{\sigma} G(\xi) d m(\xi)-\frac{1}{\left|\triangle_{L, \sigma}\right|} \int_{\triangle_{L, \sigma}} G(x) d x\right) \cdot \mathbf{n}_{K, \sigma} .
\end{aligned}
$$

Hence, $X \rightarrow X^{2}$ being convex, Lemma 7.2 gives $C_{5}$ only depending on $(N, \alpha)$ such that

$$
M_{K, \sigma}^{2} \leqslant C_{5} \frac{d_{K, \sigma}}{d_{\sigma}} \frac{h_{K}^{2}}{\left|\triangle_{K, \sigma}\right|} \int_{\triangle_{K, \sigma}}|\nabla G(x)|^{2} d x+C_{5} \frac{d_{L, \sigma}}{d_{\sigma}} \frac{h_{L}^{2}}{\left|\triangle_{L, \sigma}\right|} \int_{\triangle_{L, \sigma}}|\nabla G(x)|^{2} d x .
$$


But $\left|\triangle_{K, \sigma}\right|=m(\sigma) d_{K, \sigma} / N$ and $\left|\triangle_{L, \sigma}\right|=m(\sigma) d_{L, \sigma} / N$, so that

$$
m(\sigma) d_{\sigma} M_{K, \sigma}^{2} \leqslant C_{5} N\left(h_{K}^{2} \int_{\triangle_{K, \sigma}}|\nabla G(x)|^{2} d x+h_{L}^{2} \int_{\triangle_{L, \sigma}}|\nabla G(x)|^{2} d x\right) .
$$

Notice that, suppressing the term involving $L$, this estimate is still true if $\sigma \in \mathscr{E}_{K} \cap$ $\mathscr{E}_{\text {ext }}$.

We now gather (4.9), (4.11), (4.12) and the estimates of Proposition 4.1 in (4.8); using Hypothesis (1.4), we find thus $C_{6}$ only depending on

$$
\left(\Omega,\|\mathbf{v}\|_{\left(L^{\infty}(\Omega)\right)^{N}},\|b\|_{L^{\infty}(\Omega)}, \zeta, \alpha\right)
$$

such that

$$
\begin{aligned}
\left\|e_{\mathscr{T}}\right\|_{1, \mathscr{T}} & \\
\leqslant & C_{6} h_{\mathscr{T}}\left(\sum_{K \in \mathscr{T}} \int_{K}|\nabla \bar{u}(x)|^{2} d x\right)^{1 / 2} \\
& +C_{6} h_{\mathscr{T}}\left(\sum_{\sigma \in \mathscr{E}} \sum_{L \in \mathscr{T} \mid \sigma \in \mathscr{E}_{L}} \int_{L}\left|D^{2} \bar{u}(x)\right|^{2} d x+\int_{L}|\nabla \bar{u}(x)|^{2} d x\right. \\
& \left.\quad+\int_{L}|\nabla G(x)|^{2} d x\right)^{1 / 2} \\
\leqslant & C_{6} h_{\mathscr{T}}|||\nabla \bar{u}| \|_{L^{2}(\Omega)} \\
& +C_{6} h_{\mathscr{T}}\left(M \sum_{L \in \mathscr{T}} \int_{L}\left|D^{2} \bar{u}(x)\right|^{2} d x+\int_{L}|\nabla \bar{u}(x)|^{2} d x+\int_{L}|\nabla G(x)|^{2} d x\right)^{1 / 2} \\
\leqslant & C_{6} h_{\mathscr{T}}\||\nabla \bar{u}|\|_{L^{2}(\Omega)}+\sqrt{M} C_{6} h_{\mathscr{T}}\left(\int_{\Omega}\left|D^{2} \bar{u}(x)\right|^{2}+|\nabla \bar{u}(x)|^{2}+|\nabla G(x)|^{2} d x\right)^{1 / 2}
\end{aligned}
$$

and the proof is concluded.

\section{PROOF OF THE MAIN RESULT}

We can now prove, using interpolation techniques, Theorem 2.1.

\section{Proof of Theorem 2.1. Let}

$B=\left\{(\bar{u}, G, f) \in H_{0}^{1}(\Omega) \times\left(L^{2}(\Omega)\right)^{N} \times L^{2}(\Omega) \mid \Delta \bar{u}-\operatorname{div}(\mathbf{v} \bar{u})-b \bar{u}+\operatorname{div}(G)+f=0\right\}$ (endowed with the norm of $H_{0}^{1}(\Omega) \times\left(L^{2}(\Omega)\right)^{N} \times L^{2}(\Omega)$ ) and define $T: B \rightarrow X(\mathscr{T})$ by $T(\bar{u}, G, f)=\left(\bar{u}_{K}-u_{K}\right)_{K \in \mathscr{T}}$, where $\left(u_{K}\right)_{K \in \mathscr{T}}$ is the solution to (1.9)-(1.10). 
Corollary 3.1 shows that, if $X(\mathscr{T})$ is endowed with the discrete $H^{1}$-norm, $T$ is linear and continuous with a norm bounded by $C_{0}\left(\Omega,\|\mathbf{v}\|_{\left(L^{\infty}(\Omega)\right)^{N}}, \alpha, M\right)$.

If we define

$$
\begin{aligned}
A= & \left\{(\bar{u}, G, f) \in\left(H^{2}(\Omega) \cap H_{0}^{1}(\Omega)\right) \times\left(H^{1}(\Omega)\right)^{N} \times L^{2}(\Omega) \mid\right. \\
& \Delta \bar{u}-\operatorname{div}(\mathbf{v} \bar{u})-b \bar{u}+\operatorname{div}(G)+f=0\}
\end{aligned}
$$

(endowed with the norm of $\left(H^{2}(\Omega) \cap H_{0}^{1}(\Omega)\right) \times\left(H^{1}(\Omega)\right)^{N} \times L^{2}(\Omega)$ ), Theorem 4.1 shows that, $X(\mathscr{T})$ still being endowed with the discrete $H^{1}$-norm, $T: A \rightarrow$ $X(\mathscr{T})$ is continuous with a norm bounded by $C_{1} h_{\mathscr{T}}$, where $C_{1}$ only depends on $\left(\Omega,\|\mathbf{v}\|_{\left(L^{\infty}(\Omega)\right)^{N}},\|b\|_{L^{\infty}(\Omega)}, \zeta, \alpha, M\right)$.

Thus, by classical interpolation results (see e.g. [1], Theorem 4.1.2, p.88), $T$ is linear continuous $[A, B]_{1-s} \rightarrow X(\mathscr{T})$ with a norm bounded by

$$
C_{0}^{1-s}\left(C_{1} h_{\mathscr{T}}\right)^{s} \leqslant \max \left(C_{0}, 1\right) \max \left(C_{1}, 1\right) h_{\mathscr{T}}^{s} .
$$

Subsection 7.2 in the Appendix shows that

$$
\begin{aligned}
{[A, B]_{1-s}=} & \left\{(\bar{u}, G, f) \in\left[H^{2}(\Omega) \cap H_{0}^{1}(\Omega), H_{0}^{1}(\Omega)\right]_{1-s} \times\left(H^{s}(\Omega)\right)^{N} \times L^{2}(\Omega) \mid\right. \\
& \Delta \bar{u}-\operatorname{div}(\mathbf{v} \bar{u})-b \bar{u}+\operatorname{div}(G)+f=0\}
\end{aligned}
$$

(with equivalent norms). To conclude the proof of the theorem, it remains therefore to see that $\left[H^{2}(\Omega) \cap H_{0}^{1}(\Omega), H_{0}^{1}(\Omega)\right]_{1-s}=H^{1+s}(\Omega) \cap H_{0}^{1}(\Omega)$.

In the case $N=2$, i.e. if $\Omega$ is a polygonal open subset of $\mathbb{R}^{2}$, this result is proved in [2] (Theorem 3.1).

If $\Omega$ is convex, we propose the following simple proof (which do not uses the fact that $\Omega$ is polygonal). First of all, notice that we have, by definition, $\left[H^{2}(\Omega) \cap\right.$ $\left.H_{0}^{1}(\Omega), H_{0}^{1}(\Omega)\right]_{1-s} \hookrightarrow H_{0}^{1}(\Omega)$ and, since the inclusions

$$
H^{2}(\Omega) \cap H_{0}^{1}(\Omega) \hookrightarrow H^{2}(\Omega) \quad \text { and } \quad H_{0}^{1}(\Omega) \hookrightarrow H^{1}(\Omega)
$$

are continuous, by interpolation, there is a continuous inclusion

$$
\left[H^{2}(\Omega) \cap H_{0}^{1}(\Omega), H_{0}^{1}(\Omega)\right]_{1-s} \hookrightarrow\left[H^{2}(\Omega), H^{1}(\Omega)\right]_{1-s}=H^{1+s}(\Omega) .
$$

This shows that $\left[H^{2}(\Omega) \cap H_{0}^{1}(\Omega), H_{0}^{1}(\Omega)\right]_{1-s} \hookrightarrow H^{1+s}(\Omega) \cap H_{0}^{1}(\Omega)$.

To prove the reverse inclusion, denote $S=\Delta^{-1}$ with Dirichlet boundary conditions. Since $\Omega$ is convex, $S$ is linear continuous

$$
H^{-1}(\Omega) \rightarrow H_{0}^{1}(\Omega) \quad \text { and } \quad L^{2}(\Omega) \rightarrow H^{2}(\Omega) \cap H_{0}^{1}(\Omega) .
$$

$\Delta$ being linear continuous

$$
H^{1}(\Omega) \rightarrow H^{-1}(\Omega) \quad \text { and } \quad H^{2}(\Omega) \rightarrow L^{2}(\Omega),
$$


we deduce that $S \circ \Delta$ is linear continuous

$$
H^{1}(\Omega) \rightarrow H_{0}^{1}(\Omega) \quad \text { and } \quad H^{2}(\Omega) \rightarrow H^{2}(\Omega) \cap H_{0}^{1}(\Omega) .
$$

By interpolation, $S \circ \Delta$ is thus linear continuous

$$
\left[H^{2}(\Omega), H^{1}(\Omega)\right]_{1-s}=H^{1+s}(\Omega) \rightarrow\left[H^{2}(\Omega) \cap H_{0}^{1}(\Omega), H_{0}^{1}(\Omega)\right]_{1-s} .
$$

But $S \circ \Delta=I d$ on $H_{0}^{1}(\Omega)$, and this shows therefore that $H^{1+s}(\Omega) \cap H_{0}^{1}(\Omega)$ is continuously imbedded in $\left[H^{2}(\Omega) \cap H_{0}^{1}(\Omega), H_{0}^{1}(\Omega)\right]_{1-s}$, which concludes the proof of the theorem.

\section{NUMERICAL RESULTS}

We present here a few numerical results which illustrate the convergence results we have just proved.

In all these tests, the open set is $\Omega=]-1,1[2$ and we have taken no lower order term, i.e. $\mathbf{v}=0$ and $b=0$ in (1.1); as a right-hand side, we have let $f=0$ and $G=$ $-\nabla u$ (some tests have also been made with $G=-\nabla u+\mathbf{W}$ where $\mathbf{W}$ is divergence free, and the results are similar, provided that $\mathbf{W}$ has the required regularity). The meshes used are regular cartesian grids, and we analyse the rate of convergence by showing, in each case, the discrete $H^{1}$ norm of the error versus the size of the mesh, in log-log scale.

Our first test function is a pyramid, based on the function $(x, y) \rightarrow(1-|x|)(1-$ $|y|)$ that we have twisted in order that the peak be at $(1 / \sqrt{2}, 1 / \sqrt{2})$ instead of $(0,0)$ (this has been done to avoid too good convergence results due to symetries between the function and the mesh). The results are shown in Figure 1. The dots on this figure indicates a reference slope; as we can see, the rate of convergence is roughly 0.5 , which is the expected result since the function is here in $H^{3 / 2-\varepsilon}$ for all $\varepsilon>0$.

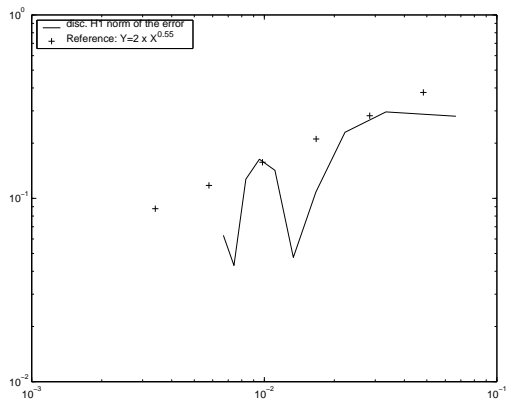

Figure 1. "Pyramid function"; reference slope: 0.55

Then, we have taken $(x, y) \rightarrow\left(1-x^{2}\right)\left(1-y^{2}\right)|(x, y)|^{s}$, which belongs (if $s \in$ ]0,1[) to $H^{1+s-\varepsilon}$ for all $\varepsilon>0$. Figure 2 shows different cases for $s$ which confirm the result of Theorem 2.1 . 

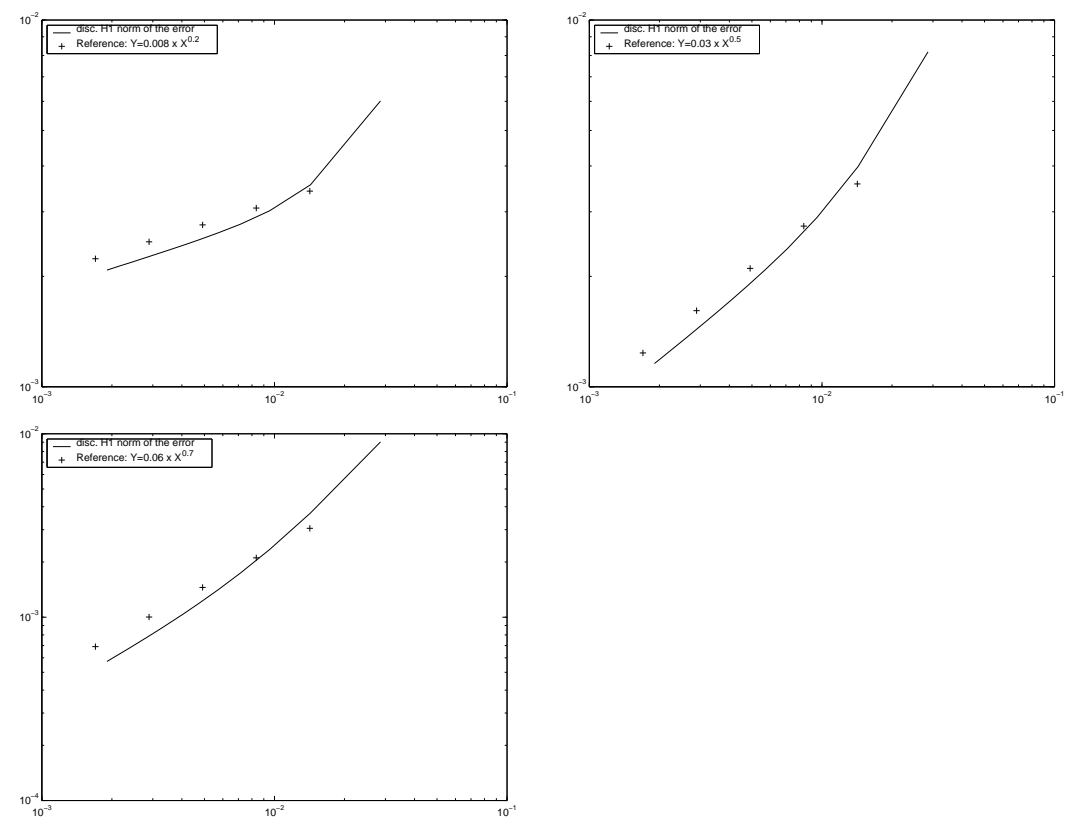

Figure 2. $|(x, y)|^{s}$ singularity; cases $s=0.2$ (reference slope: 0.2 ), $s=0.5$ (reference slope: 0.5 ), $s=0.7$ (reference slope: 0.7 )

If we add a convection term, with negative divergence, which provokes the loss of coercivity in (1.1), the results are similar to the preceding ones, the only difference being that the constant " $\mathrm{C}$ " appearing in Theorem 2.1 is much bigger. For example, coming back to the first test function, the constant in the reference slope of figure 1 is 2 whereas, if we add a convection term with $\mathbf{v}=-10(x, y)$, it becomes 80 (and the slope does not change).

\section{APPENDIX}

\subsection{Technical lemmas}

Lemma 7.1. There exists $C>0$ only depending on $N$ such that, if $U$ and $V$ are non-empty open subsets of $\mathbb{R}^{N}$ contained in a same ball of radius $R$, we have, for all $v \in H^{1}(\operatorname{co}(U \cup V))$,

$$
\begin{aligned}
& \left|\frac{1}{|U|} \int_{U} v(x) d x-\frac{1}{|V|} \int_{V} v(y) d y\right|^{2} \\
& \quad \leqslant\left(\frac{1}{|U||V|} \int_{U} \int_{V}|v(x)-v(y)| d x d y\right)^{2} \leqslant \frac{C R^{N+2}}{|U||V|} \int_{\operatorname{co}(U \cup V)}|\nabla v(z)|^{2} d z .
\end{aligned}
$$

Proof of Lemma 7.1. $\operatorname{co}(U \cup V)$ is a convex open set of $\mathbb{R}^{N}$. Thus, its boundary 
is Lipschitz-continuous and the regular functions are dense in $H^{1}(\operatorname{co}(U \cup V))$. We therefore just have to prove the lemma for regular functions.

The first inequality is obvious. Let us prove the second. If $v$ is regular, we have, for all $x \in U$ and all $y \in V, v(x)-v(y)=\int_{0}^{1} \nabla v(t x+(1-t) y) \cdot(x-y) d t$. This implies

$\frac{1}{|U||V|} \int_{U} \int_{V}|v(x)-v(y)| d x d y \leqslant \frac{1}{|U||V|} \int_{U} \int_{V} \int_{0}^{1}|\nabla v(t x+(1-t) y)||x-y| d t d y d x$

and, since $|x-y| \leqslant 2 R$ for all $x \in U$ and all $y \in V$ ( $U$ and $V$ are contained in a same ball of radius $R$ ), Jensen's inequality gives

$$
\begin{aligned}
& \left(\frac{1}{|U||V|} \int_{U} \int_{V}|v(x)-v(y)| d x d y\right)^{2} \\
& \quad \leqslant \frac{4 R^{2}}{|U||V|} \int_{U} \int_{V} \int_{0}^{1}|\nabla v(t x+(1-t) y)|^{2} d t d y d x .
\end{aligned}
$$

Let $y \in V$. Using the change of variable $x \in U \rightarrow z=t x+(1-t) y \in t U+(1-$ $t) y \subset \operatorname{co}(U \cup V)$ and Fubini's theorem, we find

$$
\int_{U} \int_{V} \int_{0}^{1}|\nabla v(t x+(1-t) y)|^{2} d t d x d y \leqslant \int_{\operatorname{co}(U \cup V)}|\nabla v(z)|^{2} \int_{V} \int_{I(z, y)} t^{-N} d t d y d z
$$

where $I(z, y)=\{t \in[0,1] \mid \exists x \in U, t x+(1-t) y=z\}$. If $t \in I(z, y)$, then $t(x-y)=$ $z-y$ for some $x \in U$; since $U$ and $V$ are contained in a same ball of radius $R$, we have then $2 R t \geqslant t|x-y|=|z-y|$ and thus $I(z, y) \subset\left[\frac{|z-y|}{2 R}, 1\right]$. We deduce that

$$
\int_{I(z, y)} t^{-N} d t \leqslant \int_{\frac{|z-y|}{2 R}}^{1} t^{-N} d t \leqslant \frac{1}{N-1} \frac{(2 R)^{N-1}}{|z-y|^{N-1}} .
$$

Thus, there exists $C_{0}$ only depending on $N$ such that, for all $z \in \operatorname{co}(U \cup V)$,

$$
\int_{V} \int_{I(z, y)} t^{-N} d t d y \leqslant C_{0} R^{N-1} \int_{V} \frac{1}{|z-y|^{N-1}} d y=C_{0} R^{N-1} \int_{z-V} \frac{1}{|\xi|^{N-1}} d \xi .
$$

$U$ and $V$ are included in a same ball of radius $R$; thus, $\operatorname{co}(U \cup V)$ is also included in this ball and, for all $z \in \operatorname{co}(U \cup V), z-V$ is therefore contained in $B(0,2 R)$, which allows to write, using polar coordinates,

$$
\begin{aligned}
\int_{V} \int_{I(z, y)} t^{-N} d t d y & \leqslant C_{0} R^{N-1} \int_{B(0,2 R)} \frac{1}{|\xi|^{N-1}} d \xi \\
& =C_{0} R^{N-1} C_{1} \int_{0}^{2 R} \frac{1}{\rho^{N-1}} \rho^{N-1} d \rho \\
& =2 C_{0} C_{1} R^{N}
\end{aligned}
$$

where $C_{1}$ is the $(N-1)$-dimensional measure of $\partial B(0,1)\left(C_{1}\right.$ only depends on $\left.N\right)$.

Gathering this last inequality, (7.2) and (7.1), we conclude the proof of the lemma. 
Lemma 7.2. If $\mathscr{T}$ is an admissible mesh which satisfies Hypothesis (1.3), there exists $C$ only depending on $(N, \alpha)$ such that, if $v \in H^{1}(\Omega)$, then, for all $K \in \mathscr{T}$ and all $\sigma \in \mathscr{E}_{K}$,

$$
\left|\frac{1}{\left|\triangle_{K, \sigma}\right|} \int_{\triangle_{K, \sigma}} v(x) d x-\frac{1}{m(\sigma)} \int_{\sigma} v(\xi) d m(\xi)\right|^{2} \leqslant \frac{C d_{K, \sigma}^{2}}{\left|\triangle_{K, \sigma}\right|} \int_{\triangle_{K, \sigma}}|\nabla v(x)|^{2} d x
$$

Proof of Lemma 7.2. The regular functions being dense in $H^{1}(\Omega)$, it is sufficient to prove the lemma for $v \in C^{1}\left(\mathbb{R}^{N}\right)$.

By translation and rotation, we can suppose that $\sigma=\{0\} \times \widetilde{\sigma}$ with $\widetilde{\sigma} \subset \mathbb{R}^{N-1}$ and that $x_{K}=\left(d_{K, \sigma}, 0\right)$.

For $a \in\left[0, d_{K, \sigma}\right]$, we denote $\widetilde{\sigma}_{a}=\left\{y \in \mathbb{R}^{N-1} \mid(a, y) \in \triangle_{K, \sigma}\right\}$. By definition, $(a, y) \in \triangle_{K, \sigma}$ if and only if there exists $t \in[0,1]$ and $z \in \widetilde{\sigma}$ such that $t\left(d_{K, \sigma}, 0\right)+(1-$ $t)(0, z)=(a, y)$; this is equivalent to $t=a / d_{K, \sigma}$ and $y=(1-t) z=\left(1-a / d_{K, \sigma}\right) z$. Thus, $\widetilde{\sigma}_{a}=\left(1-a / d_{K, \sigma}\right) \widetilde{\sigma}$.

For all $y \in \widetilde{\sigma}$ and all $a \in\left[0, d_{K, \sigma}\right]$, we have

$$
v(0, y)-v\left(a,\left(1-\frac{a}{d_{K, \sigma}}\right) y\right)=\int_{0}^{1} \nabla v\left(t a,\left(1-t \frac{a}{d_{K, \sigma}}\right) y\right) \cdot\left(-a, \frac{a}{d_{K, \sigma}} y\right) d t .
$$

Integrating on $y \in \widetilde{\sigma}$ and using the change of variable $z=\left(1-a / d_{K, \sigma}\right) y$, we find

$$
\begin{aligned}
& \int_{\sigma} v(\xi) d m(\xi)-\frac{1}{\left(1-\frac{a}{d_{K, \sigma}}\right)^{N-1}} \int_{\widetilde{\sigma}_{a}} v(a, z) d z \\
& =\int_{\widetilde{\sigma}} \int_{0}^{1} \nabla v\left(t a,\left(1-t \frac{a}{d_{K, \sigma}}\right) y\right) \cdot\left(-a, \frac{a}{d_{K, \sigma}} y\right) d t d y .
\end{aligned}
$$

Multiplying by $\left(1-a / d_{K, \sigma}\right)^{N-1}$ and integrating on $a \in\left[0, d_{K, \sigma}\right]$, we obtain

$$
\begin{aligned}
\int_{\sigma} v(\xi) d m(\xi) & \int_{0}^{d_{K, \sigma}}\left(1-\frac{a}{d_{K, \sigma}}\right)^{N-1} d a-\int_{0}^{d_{K, \sigma}} \int_{\widetilde{\sigma}_{a}} v(a, z) d z d a \\
=\int_{0}^{d_{K, \sigma}} & {\left[\left(1-\frac{a}{d_{K, \sigma}}\right)^{N-1} \times\right.} \\
& \left.\int_{\widetilde{\sigma}} \int_{0}^{1} \nabla v\left(t a,\left(1-t \frac{a}{d_{K, \sigma}}\right) y\right) \cdot\left(-a, \frac{a}{d_{K, \sigma}} y\right)\right] d t d y d a .
\end{aligned}
$$

But $\int_{0}^{d_{K, \sigma}}\left(1-a / d_{K, \sigma}\right)^{N-1} d a=d_{K, \sigma} / N$ and $\left|\triangle_{K, \sigma}\right|=m(\sigma) d_{K, \sigma} / N$; therefore, (7.3) 
gives, thanks to Fubini's theorem,

$$
\begin{aligned}
\frac{\left|\triangle_{K, \sigma}\right|}{m(\sigma)} \int_{\sigma} v(\xi) d m(\xi)-\int_{\triangle_{K, \sigma}} v(x) d x \\
=\int_{0}^{d_{K, \sigma}}\left[\left(1-\frac{a}{d_{K, \sigma}}\right)^{N-1} \times\right. \\
\left.\quad \int_{\widetilde{\sigma}} \int_{0}^{1} \nabla v\left(t a,\left(1-t \frac{a}{d_{K, \sigma}}\right) y\right) \cdot\left(-a, \frac{a}{d_{K, \sigma}} y\right)\right] d t d y d a .
\end{aligned}
$$

By definition of an admissible mesh, the straight line going through $x_{K}=$ $\left(d_{K, \sigma}, 0\right)$ and orthogonal to $\sigma \subset\{0\} \times \mathbb{R}^{N-1}$ intersects $\sigma$; this means that $0 \in \widetilde{\sigma}$. Moreover, $\sigma$ is contained in $\bar{K}$ which has diameter $h_{K}$; thus, $\widetilde{\sigma}$ has diameter less than or equal to $h_{K}$. By Hypothesis (1.3), $d_{K, \sigma}=\operatorname{dist}\left(x_{K}, \sigma\right) \geqslant \operatorname{dist}\left(x_{K}, \partial K\right) \geqslant \alpha h_{K}$.

We deduce that, for all $y \in \widetilde{\sigma},|y|=|y-0| \leqslant \operatorname{diam}(\widetilde{\sigma}) \leqslant h_{K} \leqslant \frac{1}{\alpha} d_{K, \sigma}$. Thus,

$$
\begin{aligned}
& \mid \int_{0}^{d_{K, \sigma}}\left[\left(1-\frac{a}{d_{K, \sigma}}\right)^{N-1} \times\right. \\
&\left.\int_{\tilde{\sigma}} \int_{0}^{1} \nabla v\left(t a,\left(1-t \frac{a}{d_{K, \sigma}}\right) y\right) \cdot\left(-a, \frac{a}{d_{K, \sigma}} y\right)\right] d t d y d a \mid \\
& \leqslant C_{0} \int_{0}^{d_{K, \sigma}}\left[\left(1-\frac{a}{d_{K, \sigma}}\right)^{N-1} \times\right. \\
&\left.\int_{\tilde{\sigma}} \int_{0}^{1}\left|\nabla v\left(t a,\left(1-t \frac{a}{d_{K, \sigma}}\right) y\right)\right|\right] \text { adtdyda }
\end{aligned}
$$

where $C_{0}$ only depends on $\alpha$.

Let $a \in] 0, d_{K, \sigma}[$. By the change of variable

$$
\left.\varphi_{a}:(t, y) \in\right] 0,1\left[\times \widetilde{\sigma} \rightarrow z=\left(t a,\left(1-t \frac{a}{d_{K, \sigma}}\right) y\right) \in \varphi_{a}(] 0,1[\times \widetilde{\sigma})\right.
$$

(whose Jacobian determinant is $a\left(1-t a / d_{K, \sigma}\right)^{N-1}=a\left(1-z_{1} / d_{K, \sigma}\right)^{N-1}$ since $z_{1}=$ $t a$ ), we have

$$
\begin{aligned}
\int_{\widetilde{\sigma}} \int_{0}^{1}\left|\nabla v\left(t a,\left(1-t \frac{a}{d_{K, \sigma}}\right) y\right)\right| d t d y \\
\quad=\int_{\varphi_{a}(00,1[\times \widetilde{\sigma})}|\nabla v(z)| a^{-1}\left(1-\frac{z_{1}}{d_{K, \sigma}}\right)^{-N+1} d z .
\end{aligned}
$$

But, since $\left(t a,\left(1-t \frac{a}{d_{K, \sigma}}\right) y\right)=\frac{t a}{d_{K, \sigma}}\left(d_{K, \sigma}, 0\right)+\left(1-\frac{t a}{d_{K, \sigma}}\right)(0, y)$, we have

$$
\varphi_{a}(] 0,1[\times \tilde{\sigma}) \subset \triangle_{K, \sigma}
$$


and Fubini's theorem allows thus to write

$$
\begin{aligned}
& \int_{0}^{d_{K, \sigma}}\left(1-\frac{a}{d_{K, \sigma}}\right)^{N-1} \int_{\widetilde{\sigma}} \int_{0}^{1}\left|\nabla v\left(t a,\left(1-t \frac{a}{d_{K, \sigma}}\right) y\right)\right| a d t d y d a \\
& =\int_{0}^{d_{K, \sigma}} \int_{\varphi_{a}(] 0,1[\times \widetilde{\sigma})}|\nabla v(z)|\left(1-\frac{z_{1}}{d_{K, \sigma}}\right)^{-N+1}\left(1-\frac{a}{d_{K, \sigma}}\right)^{N-1} d z d a \\
& \quad \leqslant \int_{\triangle_{K, \sigma}}|\nabla v(z)| \int_{a \in\left[0, d_{K, \sigma}\right] \mid z \in \varphi_{a}(] 0,1[\times \widetilde{\sigma})}\left(\frac{1-\frac{a}{d_{K, \sigma}}}{1-\frac{z_{1}}{d_{K, \sigma}}}\right)^{N-1} d a d z .
\end{aligned}
$$

If $z \in \varphi_{a}(] 0,1[\times \tilde{\sigma})$, we have $z_{1}=t a$ for some $\left.t \in\right] 0,1\left[\right.$, i.e. $0 \leqslant z_{1} \leqslant a$. Therefore, $1-\frac{a}{d_{K, \sigma}} \leqslant 1-\frac{z_{1}}{d_{K, \sigma}}$ and

$$
\int_{a \in\left[0, d_{K, \sigma}\right] \mid z \in \varphi_{a}(] 0,1[\times \widetilde{\sigma})}\left(\frac{1-\frac{a}{d_{K, \sigma}}}{1-\frac{z_{1}}{d_{K, \sigma}}}\right)^{N-1} d a \leqslant d_{K, \sigma} .
$$

We deduce that

$$
\begin{aligned}
& \int_{0}^{d_{K, \sigma}}\left(1-\frac{a}{d_{K, \sigma}}\right)^{N-1} \int_{\widetilde{\sigma}} \int_{0}^{1}\left|\nabla v\left(t a,\left(1-t \frac{a}{d_{K, \sigma}}\right) y\right)\right| a d t d y d a \\
& \leqslant d_{K, \sigma} \int_{\triangle_{K, \sigma}}|\nabla v(z)| d z \text {. }
\end{aligned}
$$

We now use this inequality in (7.5) and introduce the resulting estimate in (7.4) to obtain

$$
\left|\frac{1}{\left|\triangle_{K, \sigma}\right|} \int_{\triangle_{K, \sigma}} v(x) d x-\frac{1}{m(\sigma)} \int_{\sigma} v(\xi) d m(\xi)\right| \leqslant \frac{C_{0} d_{K, \sigma}}{\left|\triangle_{K, \sigma}\right|} \int_{\triangle_{K, \sigma}}|\nabla v(x)| d x .
$$

Jensen's inequality concludes then the proof of the lemma.

Lemma 7.3. Let $\mathscr{T}$ be an admissible mesh, $\sigma=K \mid L \in \mathscr{E}$ int and $U=K \cup \sigma \cup L$. Then $U$ is an open subset of $\mathbb{R}^{N}$ and $C^{\infty}(\bar{U})$ is dense in $H^{2}(U)$.

Proof of Lemma 7.3. Step 1: we prove that $U$ is open.

By translation and rotation, we can suppose that $\sigma=\{0\} \times \widetilde{\sigma}$ with $\widetilde{\sigma} \subset \mathbb{R}^{N-1}$ and, since the line going through $\left(x_{K}, x_{L}\right)$ is orthogonal to $\sigma$, that $0 \in \sigma, x_{K}=(b, 0) \in$ ] $0, \infty\left[\times\{0\}\right.$ and $\left.x_{L}=(a, 0) \in\right]-\infty, 0[\times\{0\}$. Define then $\varphi:(t, y) \in]-1,1\left[\times \mathbb{R}^{N-1} \rightarrow\right.$ $\left.\left(t^{-} a+t^{+} b,(1-|t|) y\right) \in\right] a, b\left[\times \mathbb{R}^{N-1}\right.$ (where $t^{+}=\max (0, t)$ and $\left.t^{-}=\max (0,-t)\right)$; $\varphi$ is an homeomorphism (the inverse mapping is $\psi\left(z_{1}, z^{\prime}\right)=\left(\frac{z_{1}^{+}}{b}+\frac{z_{1}^{-}}{a},\left(1-\frac{z_{1}^{+}}{b}-\right.\right.$ 
$\left.\left.\left.\frac{z_{1}^{-}}{a}\right)^{-1} z^{\prime}\right)\right)$. It is easy to see that $\varphi(]-1,1[\times \tilde{\sigma})=\triangle_{K, \sigma} \cup \sigma \cup \triangle_{L, \sigma}$; indeed, $\varphi(0, \tilde{\sigma})=$ $\sigma, \varphi(] 0,1[\times \widetilde{\sigma})=\triangle_{K, \sigma}\left(\right.$ recall that $\left.x_{K}=(b, 0)\right)$ and $\varphi(]-1,0[, \tilde{\sigma})=\triangle_{L, \sigma}($ recall that $\left.x_{L}=(a, 0)\right)$; by hypothesis on the egdes, $\widetilde{\sigma}$ is open in $\mathbb{R}^{N-1}$ and $\varphi(]-1,1[\times \widetilde{\sigma})=$ $\triangle_{K, \sigma} \cup \sigma \cup \triangle_{L, \sigma}$ is thus open in $] a, b\left[\times \mathbb{R}^{N-1}\right.$, i.e. in $\mathbb{R}^{N}$.

We have $U=K \cup \sigma \cup L=K \cup \triangle_{K, \sigma} \cup \sigma \cup \triangle_{L, \sigma} \cup L$ (because $\triangle_{K, \sigma} \subset K$ and $\triangle_{L, \sigma} \subset L$ by convexity of $K$ and $L$ ), and this proves that $U$ is open in $\mathbb{R}^{N}$ ( $K$ and $L$ are open in $\mathbb{R}^{N}$ ).

Step 2: we prove that, for all $\lambda>1, \bar{U} \subset \lambda U$ (recall that $0 \in \sigma \subset U$ ).

To see this, it is sufficient to show that, for all $z \in \bar{U},] 0, z[\subset U$; indeed, once we have obtained this result, we write, for $z \in \bar{U} \backslash\{0\}$ (the case $z=0$ is obvious) and $\lambda>1, z=\lambda\left(\frac{1}{\lambda} z\right)$ and, since $\left.\frac{1}{\lambda} z=\frac{1}{\lambda} z+\left(1-\frac{1}{\lambda}\right) \times 0 \in\right] 0, z\left[\subset U\right.$ (because $\left.\frac{1}{\lambda} \in\right] 0,1[$ ), we deduce that $z \in \lambda U$.

Let us take $z \in \bar{U}=\bar{L} \cup \bar{\sigma} \cup \bar{K}$.

Assume first that $z \in \bar{\sigma}$. Then, since $\sigma$ is an open convex subset of $\{0\} \times \mathbb{R}^{N-1}$ and $0 \in \sigma$, a classical convexity lemma tells us that $] 0, z[\subset \sigma \subset U$, which concludes this case.

Assume now that $z \in \bar{K} \backslash \bar{\sigma}$ (the case $z \in \bar{L} \backslash \bar{\sigma}$ being treated the same way).

If $z \in K$, then by the same convexity lemma as before, since $0 \in \bar{K}$ and $K$ is convex and open, we have $] 0, z[\subset K \subset U$. We can thus suppose that $z \in \partial K \backslash \bar{\sigma}$.

Let us stop a moment to prove the following geometrical fact: $\partial K \cap(\{0\} \times$ $\left.\mathbb{R}^{N-1}\right)=\bar{\sigma}$.

We first notice that $K \cap\left(\{0\} \times \mathbb{R}^{N-1}\right)=\emptyset$ : indeed, if it is not the case, then, taking $a \in K \cap\left(\{0\} \times \mathbb{R}^{N-1}\right)$, since $0 \in \bar{K}$, we have $\left.] 0, a\right] \subset K$; but $0 \in \sigma$ which is open in $\{0\} \times \mathbb{R}^{N-1}$ and, since $\left.] 0, a\right] \subset\{0\} \times \mathbb{R}^{N-1}$ with $a \neq 0$ (because $0 \in \partial K$, which does not intersects $K$ ), we can find $b \in] 0, a] \cap \sigma$; this means that $b \in \partial K \cap K$, which is not possible.

Thus, $A:=\partial K \cap\left(\{0\} \times \mathbb{R}^{N-1}\right)$ is equal to $\bar{K} \cap\left(\{0\} \times \mathbb{R}^{N-1}\right)$ and is therefore convex (because $\bar{K}$ and $\{0\} \times \mathbb{R}^{N-1}$ are convex).

We have $\sigma \subset A$ (and thus $\bar{\sigma} \subset A, A$ being closed); take $c \in A \subset\{0\} \times \mathbb{R}^{N-1}$. Since $A$ is convex, the set $O=\cup_{0 \leqslant t<1}(t c+(1-t) \sigma)$ is contained in $A$; we want to show that $O \backslash \bar{\sigma}=\emptyset$.

$\sigma$ being open in $\{0\} \times \mathbb{R}^{N-1}, O$ and thus $O \backslash \bar{\sigma}$ are also open in $\{0\} \times \mathbb{R}^{N-1}$. We have $O \backslash \bar{\sigma} \subset \partial K$, which implies $O \backslash \bar{\sigma} \subset \cup_{\left\{\sigma^{\prime} \in \mathscr{E}_{K}, \sigma^{\prime} \neq \sigma\right\}} \overline{\sigma^{\prime}}$. Suppose that $O \backslash \bar{\sigma}$ is not empty; then, since it is an open subset of $\{0\} \times \mathbb{R}^{N-1}$ and $\left\{\sigma^{\prime} \in \mathscr{E}_{K}, \sigma^{\prime} \neq \sigma\right\}$ is finite, there exists $\sigma^{\prime} \in \mathscr{E}_{K} \backslash\{\sigma\}$ whose adherence contains $N$ points of $O \backslash \bar{\sigma} \subset\{0\} \times$ $\mathbb{R}^{N-1}$ in general position. Thus, the hyperplane containing $\sigma^{\prime}$ is the affine space generated by these points, that is to say $\{0\} \times \mathbb{R}^{N-1}$. By hypothesis on the edges, the intersection of $\{0\} \times \mathbb{R}^{N-1}$ and of the line going through $x_{K}$ and orthogonal to $\{0\} \times \mathbb{R}^{N-1}$ belongs to both $\sigma$ and $\sigma^{\prime}$ (since $\sigma$ and $\sigma^{\prime}$ both generate $\{0\} \times \mathbb{R}^{N-1}$ ), which is a contradiction with the fact that $\sigma$ and $\sigma^{\prime}$ are disjoint. 
Thus, $O \backslash \bar{\sigma}$ is empty, and $O \subset \bar{\sigma}$. Since $c \in \bar{O}$, we deduce that $c \in \bar{\sigma}$, and this concludes the proof that $A \subset \bar{\sigma}$, i.e. that $\partial K \cap\left(\{0\} \times \mathbb{R}^{N-1}\right)=\bar{\sigma}$.

We also notice that, since $K \cap\left(\{0\} \times \mathbb{R}^{N-1}\right)=\emptyset$ (see above) and since $x_{K} \in$ ] $0, \infty\left[\times \mathbb{R}^{N-1}\right.$, by connexity of $K$, we have $\left.K \subset\right] 0, \infty\left[\times \mathbb{R}^{N-1}\right.$. We prove the same way that $L \subset]-\infty, 0\left[\times \mathbb{R}^{N-1}\right.$.

Let us now return to the proof that, if $z \in \partial K \backslash \bar{\sigma}$, then $] 0, z[\subset U$.

As we have seen before, $\triangle_{K, \sigma} \cup \sigma \cup \triangle_{L, \sigma}$ is an open set and, since 0 belongs to this open set (and $z \neq 0),] 0, z\left[\cap\left(\triangle_{K, \sigma} \cup \sigma \cup \triangle_{L, \sigma}\right)\right.$ is not empty. We have $z \in$ $\bar{K} \subset\left[0, \infty\left[\times \mathbb{R}^{N-1}\right.\right.$; since $z \in \partial K \backslash \bar{\sigma}$ and $\partial K \cap\left(\{0\} \times \mathbb{R}^{N-1}\right)=\bar{\sigma}$, this implies $z \notin$ $\{0\} \times \mathbb{R}^{N-1}$ and thus $\left.z \in\right] 0, \infty\left[\times \mathbb{R}^{N-1}\right.$. Hence, $] 0, z[\subset] 0, \infty\left[\times \mathbb{R}^{N-1}\right.$.

But $\left.\triangle_{L, \sigma} \subset L \subset\right]-\infty, 0\left[\times \mathbb{R}^{N-1}\right.$ and $\sigma \subset\{0\} \times \mathbb{R}^{N-1}$; therefore, $] 0, z\left[\cap\left(\triangle_{K, \sigma} \cup\right.\right.$ $\left.\left.\sigma \cup \triangle_{L, \sigma}\right)=\right] 0, z\left[\cap \triangle_{K, \sigma}\right.$. This set being non-empty, we can take $\left.c \in\right] 0, z\left[\cap \triangle_{K, \sigma} \subset K\right.$. Since 0 and $z$ belong to $\bar{K},] 0, c]$ and $[c, z[$ are contained in $K$, which implies that ] $0, z[=] 0, c] \cup[c, z[\subset K$ and concludes this step.

Step 3: we prove the density result.

Take $v \in H^{2}(U)$ and define, for $\lambda>1, v_{\lambda}(x)=v(x / \lambda)$; $v_{\lambda}$ belongs to $H^{2}(\lambda U)$ and the restriction of $v_{\lambda}$ to $U \subset \lambda U$ converges, as $\lambda \rightarrow 1$, to $v$ in $H^{2}(U)$.

Indeed, to see the convergence in $L^{2}(U)$, we take $\varepsilon>0$ and $w \in C_{c}(U)$ such that $\|v-w\|_{L^{2}(U)}<\varepsilon$; we then write, with $w_{\lambda}(x)=w(x / \lambda),\left\|v_{\lambda}-v\right\|_{L^{2}(U)} \leqslant \| v_{\lambda}-$ $w_{\lambda}\left\|_{L^{2}(U)}+\right\| w_{\lambda}-w\left\|_{L^{2}(U)}+\right\| w-v \|_{L^{2}(U)}$. By a change of variable, we have $\| v_{\lambda}-$ $w_{\lambda}\left\|_{L^{2}(U)} \leqslant\right\| v_{\lambda}-w_{\lambda}\left\|_{L^{2}(\lambda U)}=\lambda^{N / 2}\right\| v-w \|_{L^{2}(U)} \leqslant \lambda^{N / 2} \varepsilon$, so that $\left\|v_{\lambda}-v\right\|_{L^{2}(U)} \leqslant$ $\left(\lambda^{N / 2}+1\right) \varepsilon+\left\|w_{\lambda}-w\right\|_{L^{2}(U)}$. Since $w \in C_{c}(U)$, the dominated convergence theorem (for example) gives $\left\|w_{\lambda}-w\right\|_{L^{2}(U)} \rightarrow 0$ as $\lambda \rightarrow 1$ and this concludes the proof of the $L^{2}$ convergence. The first and second derivatives of $v_{\lambda}$ being (with evident notations) $\lambda^{-1}(\nabla v)_{\lambda}$ and $\lambda^{-2}\left(D^{2} v\right)_{\lambda}$, the $H^{2}$ convergence is an immediate consequence of the $L^{2}$ convergence showed above.

To approximate $v$ in $H^{2}(U)$ by regular function, we thus just need to approximate $v_{\lambda}$ in this space. We extend $v_{\lambda}$ to $\mathbb{R}^{N}$ by 0 outside $\lambda U$ and take $\left(\rho_{n}\right)_{n \geqslant 1}$ a smoothing kernel; $v_{\lambda} * \rho_{n} \in C_{c}^{\infty}\left(\mathbb{R}^{N}\right)$ and, since $U$ is relatively compact in $\lambda U$ and $v_{\lambda} \in H^{2}(\lambda U)$, we have $v_{\lambda} * \rho_{n} \rightarrow v_{\lambda}$ in $H^{2}(U)$ as $n \rightarrow \infty$ (because, for $n$ large enough - such that $U+\operatorname{supp}\left(\rho_{n}\right) \subset \lambda U-$, we have $\nabla\left(v_{\lambda} * \rho_{n}\right)=\left(\nabla v_{\lambda}\right) * \rho_{n}$ and $D^{2}\left(v_{\lambda} * \rho_{n}\right)=\left(D^{2} v_{\lambda}\right) * \rho_{n}$ on $\left.U\right)$. This concludes the proof of the lemma.

Lemma 7.4. If $U$ is a convex open bounded set in $\mathbb{R}^{N}$ and $\mathbf{W} \in(C(\bar{U}))^{N}$ is such that $\operatorname{div}(\mathbf{W}) \in L^{1}(U)$, then

$$
\int_{U} \operatorname{div}(\mathbf{W})(x) d x=\int_{\partial U} \mathbf{W}(\xi) \cdot \mathbf{n}(\xi) d m(\xi)
$$

( $m$ denotes here the $(N-1)$-dimensional measure on $\partial U$ and $\mathbf{n}$ is the unit normal to $\partial U$ outward to $U$-notice that, since $U$ is convex, it has a Lipschitz-continuous boundary). 
Proof of Lemma 7.4. By translation, we can suppose that $0 \in U$. Then, for all $z \in \bar{U}$, since $U$ is convex, $[0, z[\in U$; as we have seen in step 2 of the proof of Lemma 7.3, this implies that, for all $\lambda>1, \bar{U} \subset \lambda U$.

Let $\mathbf{W}_{\lambda}(x)=\mathbf{W}(x / \lambda)$; we have $\mathbf{W}_{\lambda} \in(C(\lambda \bar{U}))^{N}$ and $\mathbf{W}_{\lambda} \rightarrow \mathbf{W}$ uniformly on $\bar{U}$ (and thus on $\partial U$ ) as $\lambda \rightarrow 1$ (this is due to the uniform continuity of $\mathbf{W}$ on this set). Moreover, $\operatorname{div}\left(W_{\lambda}\right)=\lambda^{-1}(\operatorname{div}(\mathbf{W}))_{\lambda} \in L^{1}(\lambda U)$ and, as in step 3 of the proof of Lemma 7.3, we deduce that $\operatorname{div}\left(\mathbf{W}_{\lambda}\right) \rightarrow \operatorname{div}(\mathbf{W})$ in $L^{1}(U)$ as $\lambda \rightarrow 1$. It is thus sufficient to prove that, for all $\lambda>1, \mathbf{W}_{\lambda}$ satisfies the result of the Lemma.

Let $\left(\rho_{n}\right)_{n \geqslant 1}$ be a smoothing kernel. Extend $\mathbf{W}_{\lambda}$ to $\mathbb{R}^{N}$ by 0 outside $\lambda U$ and define $\mathbf{W}_{n, \lambda}=\mathbf{W}_{\lambda} * \rho_{n} \in C_{c}^{\infty}\left(\mathbb{R}^{N}\right)$. By regularity of $W_{n, \lambda}$, we have

$$
\int_{U} \operatorname{div}\left(\mathbf{W}_{n, \lambda}\right)(x) d x=\int_{\partial U} \mathbf{W}_{n, \lambda}(\xi) \cdot \mathbf{n}(\xi) d m(\xi) .
$$

But $\mathbf{W}_{\lambda}$ is uniformly continuous on the open set $\lambda U$, which contains the compact set $\partial U$; thus, $\mathbf{W}_{n, \lambda} \rightarrow \mathbf{W}_{\lambda}$ uniformly on $\partial U$.

We have, in the sense of the distributions on $\mathbb{R}^{N}, \operatorname{div}\left(\mathbf{W}_{n, \lambda}\right)=\operatorname{div}\left(\mathbf{W}_{\lambda}\right) * \rho_{n}$. Since $\operatorname{div}\left(\mathbf{W}_{\lambda}\right) \in L^{1}(\lambda U)$ and $U$ is relatively compact in $\lambda U$, we deduce that $\operatorname{div}\left(\mathbf{W}_{n, \lambda}\right) \rightarrow \operatorname{div}\left(\mathbf{W}_{\lambda}\right)$ in $L^{1}(U)$ as $n \rightarrow \infty$.

These convergences allow to pass to the limit in (7.6) to see that $\mathbf{W}_{\lambda}$ satisfies the result of the lemma, which concludes the proof.

\subsection{Interpolation}

We prove in this subsection that, if $\Omega$ is a bounded open subset of $\mathbb{R}^{N}(N=2$ or 3) with a Lipschitz-continous boundary and $\mathbf{v} \in(C(\bar{\Omega}))^{N}$ satisfies $\operatorname{div}(\mathbf{v}) \in L^{2}(\Omega)$, then, for all $\vartheta \in] 0,1$, the interpolate space of order $\vartheta$ between

$$
\begin{aligned}
A= & \left\{(\bar{u}, G, f) \in\left(H^{2}(\Omega) \cap H_{0}^{1}(\Omega)\right) \times\left(H^{1}(\Omega)\right)^{N} \times L^{2}(\Omega) \mid\right. \\
& \Delta \bar{u}-\operatorname{div}(\mathbf{v} \bar{u})-b \bar{u}+\operatorname{div}(G)+f=0\}
\end{aligned}
$$

and

$$
B=\left\{(\bar{u}, G, f) \in H_{0}^{1}(\Omega) \times\left(L^{2}(\Omega)\right)^{N} \times L^{2}(\Omega) \mid \Delta \bar{u}-\operatorname{div}(\mathbf{v} \bar{u})-b \bar{u}+\operatorname{div}(G)+f=0\right\}
$$

is (with equivalent norms)

$$
\begin{aligned}
C= & \left\{(\bar{u}, G, f) \in\left[H^{2}(\Omega) \cap H_{0}^{1}(\Omega), H_{0}^{1}(\Omega)\right]_{\vartheta} \times\left(H^{1-\vartheta}(\Omega)\right)^{N} \times L^{2}(\Omega) \mid\right. \\
& \Delta \bar{u}-\operatorname{div}(\mathbf{v} \bar{u})-b \bar{u}+\operatorname{div}(G)+f=0\},
\end{aligned}
$$

each of these spaces being endowed by its natural norm (notice that the interpolate space of order $\vartheta$ between $H^{1}(\Omega)$ and $L^{2}(\Omega)$ is $H^{1-\vartheta}(\Omega)$ ).

This result is quite natural, but not so easy to prove. 
To simplify the notations, we let $V=H_{0}^{1}(\Omega)$ and $W=H^{2}(\Omega) \cap H_{0}^{1}(\Omega)$. The proof relies on a result in [11]. Define the linear application

$$
T \in \mathscr{L}\left(V \times\left(L^{2}(\Omega)\right)^{N} \times L^{2}(\Omega) ; H^{-1}(\Omega)\right) \cap \mathscr{L}\left(W \times\left(H^{1}(\Omega)\right)^{N} \times L^{2}(\Omega) ; L^{2}(\Omega)\right)
$$

by $T(\bar{u}, G, f)=\Delta \bar{u}-\operatorname{div}(\mathbf{v} \bar{u})-b \bar{u}+\operatorname{div}(G)+f$; this application is continuous $W \times$ $\left(H^{1}(\Omega)\right)^{N} \times L^{2}(\Omega) \rightarrow L^{2}(\Omega)$ because, since $\operatorname{div}(\mathbf{v}) \in L^{2}(\Omega)$ - this is the only place where we need this hypothesis - and $W \subset C(\bar{\Omega})$ - recall that $N \leqslant 3-$, we have $\operatorname{div}(\mathbf{v} \bar{u})=\operatorname{div}(\mathbf{v}) \bar{u}+\mathbf{v} \cdot \nabla \bar{u} \in L^{2}(\Omega)$ when $\bar{u} \in W$. Then

$$
\begin{aligned}
& A=\left\{x \in W \times\left(H^{1}(\Omega)\right)^{N} \times L^{2}(\Omega) \mid T(x)=0\right\}, \\
& B=\left\{x \in V \times\left(L^{2}(\Omega)\right)^{N} \times L^{2}(\Omega) \mid T(x)=0\right\}
\end{aligned}
$$

and Theorem 14.3 in [11]* allows to see that

$$
[A, B]_{\vartheta}=\left\{x \in\left[W \times\left(H^{1}(\Omega)\right)^{N} \times L^{2}(\Omega), V \times\left(L^{2}(\Omega)\right)^{N} \times L^{2}(\Omega)\right]_{\vartheta} \mid T(x)=0\right\}=C
$$

with equivalent norms (notice that this last space is equal to $C$ because the interpolate space of a product of spaces is the product of the corresponding interpolate spaces), provided that we can construct an application

$$
\left.R \in \mathscr{L}\left(H^{-1}(\Omega)\right) ; V \times\left(L^{2}(\Omega)\right)^{N} \times L^{2}(\Omega)\right) \cap \mathscr{L}\left(L^{2}(\Omega) ; W \times\left(H^{1}(\Omega)\right)^{N} \times L^{2}(\Omega)\right)
$$

such that $T \circ R=I d$ on $H^{-1}(\Omega)$.

The rest of this subsection is devoted to the construction of such a $R$.

The main difficulty in constructing this application is the lack of regularity of $\partial \Omega$. If $\Omega$ is a regular (or convex) open set and $\mathbf{v} \in\left(C^{1}(\bar{\Omega})\right)^{N}$ (for example), then $R$ is quite easy to build: take, for $L \in H^{-1}(\Omega), R(L)=(\bar{u}, 0,0)$ where $\bar{u}$ is the variational solution of $\Delta \bar{u}-\operatorname{div}(\mathbf{v} \bar{u})-b \bar{u}=L$ with Dirichlet boundary conditions; the regularity of $\partial \Omega$ ensures then that $R$ is continuous $L^{2}(\Omega) \rightarrow W \times\left(H^{1}(\Omega)\right)^{N} \times L^{2}(\Omega)$.

If $\Omega$ is a polygonal non-convex open set, we must find another way to construct $R$. The main idea is to get rid of $\Omega$ and to bring ourselves back to $\mathbb{R}^{N}$.

Following an idea of [3], we first build

$$
r \in \mathscr{L}\left(L^{2}\left(\mathbb{R}^{N}\right) ; L^{2}(\Omega)\right) \cap \mathscr{L}\left(H^{1}\left(\mathbb{R}^{N}\right) ; H_{0}^{1}(\Omega)\right)
$$

such that $r(\varphi)=\varphi$ for all $\varphi \in \mathscr{D}(\Omega)$.

To do so, we notice that, since $\Omega$ has a bounded Lipschitz boundary, so does $\mathbb{R}^{N} \backslash \Omega$; there exists thus an extension operator $E$ which is continuous $H^{1}\left(\mathbb{R}^{N} \backslash \Omega\right) \rightarrow$ $H^{1}\left(\mathbb{R}^{N}\right)$ and $L^{2}\left(\mathbb{R}^{N} \backslash \Omega\right) \rightarrow L^{2}\left(\mathbb{R}^{N}\right)$ (the classical extension operators constructed

${ }^{*}$ In fact, this theorem concerns the interpolation of complex banach spaces, and we consider here spaces of real-valued functions; but it is not very difficult to see, since we handle spaces of functions, that the result of this theorem is also valid in our case of real interpolation. 
via symetries satisfy these continuities). We define $r$ by $r(\varphi)=\left(\varphi-E\left(\varphi_{\mid \mathbb{R}^{N} \backslash \Omega}\right)\right)_{\mid \Omega}$. Since $\varphi-E\left(\varphi_{\mid \mathbb{R}^{N} \backslash \Omega}\right)=0$ on $\mathbb{R}^{N} \backslash \Omega$, we clearly have $r(\varphi) \in H_{0}^{1}(\Omega)$ if $\varphi \in H^{1}\left(\mathbb{R}^{N}\right)$; moreover, if $\varphi \in \mathscr{D}(\Omega), \varphi_{\mid \mathbb{R}^{N} \backslash \Omega}=0$ so that $r(\varphi)=\varphi$, and $r$ has thus the desired properties.

$r$ allows us to extend elements of $H^{-1}(\Omega)$ into elements of $H^{-1}\left(\mathbb{R}^{N}\right)$, in such a way that elements of $L^{2}(\Omega) \subset H^{-1}(\Omega)$ are extented into elements of $L^{2}\left(\mathbb{R}^{N}\right)$.

Indeed, $r^{*}: H^{-1}(\Omega) \rightarrow H^{-1}\left(\mathbb{R}^{N}\right)$ is linear continuous and, since $r: L^{2}\left(\mathbb{R}^{N}\right) \rightarrow$ $L^{2}(\Omega)$ is continuous, $r^{*}$ is also continuous $L^{2}(\Omega) \rightarrow L^{2}\left(\mathbb{R}^{N}\right)$ (we have identified, as usual, the dual space of $L^{2}$ to $L^{2}$ itself). Moreover, if $L \in H^{-1}(\Omega)$, one has $L=r^{*}(L)$ in $\mathscr{D}^{\prime}(\Omega)$ : indeed, for all $\varphi \in \mathscr{D}(\Omega), r^{*}(L)(\varphi)=L(r(\varphi))=L(\varphi)$.

Let $L \in H^{-1}(\Omega)$; since $r^{*}(L) \in H^{-1}\left(\mathbb{R}^{N}\right)$, we can define $w^{L} \in H^{1}\left(\mathbb{R}^{N}\right)$ as the variational solution of $-\Delta w^{L}+w^{L}=r^{*}(L)$ on $\mathbb{R}^{N}$, and, since $r^{*}: H^{-1}(\Omega) \rightarrow$ $H^{-1}\left(\mathbb{R}^{N}\right)$ is linear continuous, the application $L \in H^{-1}(\Omega) \rightarrow w^{L} \in H^{1}\left(\mathbb{R}^{N}\right)$ is linear continuous. Moreover, $r^{*}$ is also linear continuous $L^{2}(\Omega) \rightarrow L^{2}\left(\mathbb{R}^{N}\right)$ so that, by the regularity properties of $-\Delta+I d$ on $\mathbb{R}^{N}, L \rightarrow w^{L}$ is linear continuous $L^{2}(\Omega) \rightarrow H^{2}\left(\mathbb{R}^{N}\right)$.

Define now $R(L)=\left(0,-\nabla\left(w_{\mid \Omega}^{L}\right), w_{\Omega}^{L}\right)$. Since the restriction to $\Omega$ is linear continuous $L^{2}\left(\mathbb{R}^{N}\right) \rightarrow L^{2}(\Omega), H^{1}\left(\mathbb{R}^{N}\right) \rightarrow H^{1}(\Omega)$ and $H^{2}\left(\mathbb{R}^{N}\right) \rightarrow H^{2}(\Omega), R$ is linear continuous $H^{-1}(\Omega) \rightarrow V \times\left(L^{2}(\Omega)\right)^{N} \times L^{2}(\Omega)$ and $L^{2}(\Omega) \rightarrow W \times\left(H^{1}(\Omega)\right)^{N} \times L^{2}(\Omega)$.

Moreover, for all $L \in H^{-1}(\Omega), T \circ R(L)=\operatorname{div}\left(-\nabla\left(w_{\mid \Omega}^{L}\right)\right)+w_{\mid \Omega}^{L}=-\Delta\left(w_{\mid \Omega}^{L}\right)+$ $w_{\mid \Omega}^{L}=r^{*}(L)_{\mid \Omega}=L$ in $\mathscr{D}^{\prime}(\Omega)$ (by properties of $r^{*}$ ), thus also in $H^{-1}(\Omega)$. This concludes the construction of $R$ and this appendix.

\section{REFERENCES}

1. J. Bergh and J. Löfström, Interpolation Spaces. Springer-Verlag, 1976.

2. C. Bacuta, J. Bramble and J. Pasciak, New interpolation results and applications to finite element methods for elliptic boundary value problems, East-West J. Numer. Math. (2001) 9, No. 3, 179198.

3. J. Bramble, Interpolation between Sobolev Spaces in Lipschitz domains with an application to multigrid theory, Math. Comp. (1995) 64, No. 212, 1359-1365.

4. J. Bramble and S. Hilbert, Bounds for a class of linear functionals with applications to Hermite interpolation, Numer. Math., (1970/1971) 16, 362-369.

5. P. Chatzipantelidis and R.D. Lazarov, The finite volume element method in nonconvex polygonal domains. In: Finite Volumes for Complex Applications III. Hermes Penton Science, Porquerolles, 2002, pp. 171-178.

6. J. Droniou, Non-coercive linear elliptic problems, Potential Analysis, (2002) 17 (2), 181-203.

7. J. Droniou J., PhD Thesis. CMI, Université de Provence Aix-Marseille I, Juin 2002. Available at http: //www-gm3. univ-mrs. fr/ droniou/these/index-en.html.

8. J. Droniou J. and T. Gallouët, Finite volume methods for convection-diffusion equations with right-hand side in $H^{-1}$, Mathematical Modelling and Numerical Analysis, (2002) 36 (4), 705724. 
9. R. Eymard, T. Gallouët and R. Herbin, Finite Volume Methods. In: Handbook of Numerical Analysis, Vol. VII, pp. 713-1020. Edited by P.G. Ciarlet and J.L. Lions (North Holland).

10. T. Gallouët, R. Herbin and M.H. Vignal, Error estimate for the approximate finite volume solutions of convection diffusion equations with Dirichlet, Neumann or Fourier boundary conditions, SIAM J. Numer. Anal., (2000) 37, No. 6, 1935-1972.

11. J-L. Lions and E. Magenes, Non-Homogeneous Boundary Value Problems and Applications, Springer-Verlag, 1972.

12. I. Mishev, Finite volume element methods for non-definite problems, Numer. Math. (1999) 83, No. 1, 161-175. 